\title{
Rank one HCIZ at high temperature: Interpolating between classical and free convolutions
}

\author{
Pierre Mergny $^{1,2 \star}$ and Marc Potters ${ }^{3}$ \\ 1 Chair of Econophysics \& Complex Systems, \\ Ecole Polytechnique, 91128 Palaiseau Cedex, France \\ 2 LPTMS, CNRS, Univ. Paris-Sud, Université Paris-Saclay, 91405 Orsay, France \\ 3 Capital Fund Management, 23 rue de l'Université, 75007 Paris, France

$$
\star \text { mergny.pierre@gmail.com }
$$

\begin{abstract}
We study the rank one Harish-Chandra-Itzykson-Zuber integral in the limit where $\frac{N \beta}{2} \rightarrow c$, called the high-temperature regime and show that it can be used to construct a promising one-parameter interpolation family, with parameter $c$ between the classical and the free convolution. This c-convolution has a simple interpretation in terms of another associated family of distribution indexed by $c$, called the Markov-Krein transform: the c-convolution of two distributions corresponds to the classical convolution of their Markov-Krein transforms. We derive first cumulant-moment relations, a central limit theorem, a Poisson limit theorem and show several numerical examples of $c$-convoluted distributions.
\end{abstract}

(c) (i) Copyright P. Mergny and M. Potters This work is licensed under the Creative Commons Attribution 4.0 International License.

Published by the SciPost Foundation.
Received 25-01-2021

Accepted 18-11-2021

Published 14-01-2022

doi:10.21468/SciPostPhys.1

\section{Contents}

1 Introduction

2 Review of some results concerning the rank one Harish-Chandra-Itzykson-Zuber Integral

2.1 A few words on the full rank case

2.2 The rank-one case

2.2.1 Power sum representation

2.2.2 Differential operator representation

2.2.3 Inverse Laplace representation

2.2.4 Spherical Dirichlet integral representation

2.2.5 Moment generating function representation

Large $N$ behavior of the rank one HCIZ

2.3.1 $\beta>0$ and relation with free probability

2.3.2 Infinite temperature regime $(\beta \rightarrow 0)$ and classical convolution 
3.1 Definition

$\begin{array}{ll}3.2 & \text { The special case } \gamma \text { even } \\ 3.3 & 14\end{array}$

$\begin{array}{ll}3.3 & \text { Link with finite free convolution } \\ \end{array}$

4 HCIZ at the high temperature limit $\frac{N \beta}{2} \rightarrow c \quad 16$

4.1 Definition and notations $\quad 16$

4.2 Generalized Stieltjes transform and fractional calculus 18

4.3 Properties of the Markov Krein transform 20

4.4 Known Markov Krein transforms $\quad 21$

4.5 Inverting the Markov-Krein transform 23

5 c-convolution $\quad 26$

5.1 c-convolution as convolution of Markov-Krein transforms 26

$\begin{array}{lll}5.2 & \text { c-cumulants } & 27\end{array}$

5.3 c-central limit theorem and related distributions 29

5.4 c-Poisson limit theorem $\quad 32$

$\begin{array}{llr}6 & \text { Conclusion } & 34\end{array}$

$\begin{array}{ll}\text { References } & 35\end{array}$

\section{Introduction}

For a self-adjoint random matrix A, of size $N$ with real, complex, or quaternionic entries, under mild assumptions and up to a rescaling of the entries, we know from Random Matrix Theory (RMT) that the (random) spectral measure of A tends to a deterministic limiting measure $\mu_{A}$ in the limit $N \rightarrow \infty$ (see for example [1]). Free Probability, introduced by Voiculescu [2], allows one to compute the limiting spectral distribution denoted by $\mu_{A} \boxplus \mu_{B}$ and known as the free convolution, for the sum of two such random matrices $\mathbf{A}$ and $\mathbf{B}$, in this limit $N \rightarrow \infty$, where one replaces the notion of independence of classical probability by the notion of freeness of non-commutative algebraic probability theory. The correspondence between classical and free probability is given in Table 1.

For a measure $\mu_{A}$ with (compact) support $I$, the transform $\mathcal{R}_{\mu_{A}}($.$) that linearizes the free$ convolution is the R-transform defined by:

$$
\mathcal{R}_{\mu_{A}}(t):=\mathcal{G}_{\mu_{A}}^{(-1)}(t)-\frac{1}{t}
$$

Table 1: Correspondence between the classical and the free world

\begin{tabular}{|c||c|}
\hline Classical Probability & Free Probability \\
\hline$X$ real random variable & A self-adjoint operator \\
Independance & Freeness \\
$\mu_{X} * \mu_{Y}$ & $\mu_{A} \boxplus \mu_{B}$ \\
$\log \mathbb{E}_{X}\left[e^{t X}\right]$ & $\mathcal{R}_{\mu_{A}}(t)$ \\
\hline
\end{tabular}


where.$^{(-1)}$ denotes the composition inverse and

$$
\mathcal{G}_{\mu_{A}}(z):=\int_{I} d x \frac{1}{z-x} \mu_{A}(x),
$$

is the Stieltjes transform. We point out that the correspondence in Table 1 is by no mean exhaustive: to cite a few missing items, there is also a clear correspondence for the multiplicative convolution with the so-called $S$-transform [1], the combinatorial moment-cumulant relations [3], the entropy [4] between the classical and the free world. Ever since the discovery of free probability, it has remain unclear whether one could find other generalized notions of independence, until Speicher [5] proved that, under specific assumptions, there are only three possible notions for a non-commutative algebraic probability space: classical independence, freeness and Boolean independence [6]. By relaxing the assumptions, it has been however possible to construct other types of convolutions see for example [7]. Despite Speicher's work, there have been several attempts to construct generalized convolutions, with or without an underlying notion of independence, that would, in particular, interpolate between the classical convolution and the free convolution. To cite a few important results, let us mention: the $q$-convolution of Nica [8] (see also [9] for a similar but different $q$-convolution) which interpolates between the classical convolution at $q=1$ and the free convolution at $q=0$ but which does not seem to preserve the positivity of the measures [10]; the $t$-convolution of BenaychGeorges and Lévy in [11], which interpolates between the classical convolution $(t=0)$ and the free convolution $(t \rightarrow \infty)$ but for which it is not possible to construct a transform that linearizes the convolution and from which one can define cumulants at any order. In this note, we construct another one-parameter convolution, called the c-convolution as a continuous interpolation between the classical convolution at $c=0$ and the free convolution as $c \rightarrow \infty$. Our construction is similar to the one developed in [8] in the sense that we construct an operator that interpolates naturally between the moment generating function and the exponential of (the integral of) the R-transform. Our c-convolution is technically defined on a set larger than the set of probability distributions and it is still an open question to know whether it preserves positivity. Nevertheless we show that several objects (see [12,13] and [14-17]) that have appeared before in the RMT literature at a specific limit, called the high temperature regime where the inverse temperature $\beta$ decays linearly with the size $N$ of the matrix $\beta \sim \frac{2 c}{N}$, admit a simple interpretation in terms of our $c$-convolution. Schematically our construction is as follows (concepts and notations will be made more precise in the main text). We start with the rank one HCIZ integral at finite $N$ and fixed $\beta$ involving a matrix with eigenvalues $\boldsymbol{a}$ and another matrix with a single non-zero eigenvalue $t$ :

$$
\mathcal{I}_{\boldsymbol{a}}^{(\beta)}(t)=\mathbb{E}_{\boldsymbol{v}}\left[e^{\left(\boldsymbol{v}^{*} \underline{\boldsymbol{a}} \boldsymbol{v}\right) t}\right]=\mathbb{E}_{X}\left[e^{X t}\right],
$$

where $v$ is the generalization to all $\beta>0$ of a unit vector with real, complex, or quaternionic entries averaged over the corresponding sphere. We have introduced the random variable $X:=\boldsymbol{v}^{*} \underline{\boldsymbol{a}} \boldsymbol{v}$ that we will call the discrete Markov-Krein transform of $\boldsymbol{a}$. The rank one HCIZ integral is then the moment generating function of this variable. As $N \rightarrow \infty$ with fixed $\beta$ the variable $X$ concentrates on its average value and the Markov-Krein transformation is not very useful, but, as we will see, the variable $X$ converges to a non-trivial measure as $N \rightarrow \infty$ with fixed $c:=N \beta / 2$. Our $c$-convolution will then be the (classical) convolution of MarkovKrein transforms, it naturally interpolates between the classical convolution $(c \rightarrow 0)$ and the free convolution $(c \rightarrow \infty)$. In Section 2, we review several results concerning this HCIZ integral in the classical regime $(\beta>0)$, that will be useful to have a better understanding of our c-convolution; we focus on the rank one HCIZ as it is our main object of study. Section 3 is technically independent of our construction of the $c$-convolution and can be read independently. There, we show that we can make sense of the HCIZ for negative values of the 
parameter $\beta$, in particular, we show that $\beta=-2$ is linked to the finite free convolution of Marcus $[18,19]$. Many of the properties of the finite free convolution will have a clear analog in the high-temperature regime. In Section 4, we define HCIZ in the high-temperature regime and derive its properties, with a particular focus on the Markov-Krein transform. Eventually, in Section 5, we introduce and discuss the properties of the $c$-convolution and derive several examples of $c$-convoluted objects.

\section{Review of some results concerning the rank one Harish-Chandra- Itzykson-Zuber Integral}

\subsection{A few words on the full rank case}

In the 80', Itzykson and Zuber re-discovered Harish-Chandra's work on integrals over Lie groups [20], in the context of random matrix theory (RMT). Such integrals are now referred to as Harish-Chandra -Itzykson-Zuber (HCIZ in short) integrals, also known in the literature as angular/spherical integrals and as multivariate Bessel function. If we denote by $\beta=1,2,4$ and A and B two $N \times N$ self-adjoint matrices with real, complex, or quaternionic entries respectively, the HCIZ reads ${ }^{1}$ :

$$
\mathcal{I}^{(\beta)}(\mathbf{A}, \mathbf{B}):=\int_{\mathbf{G} \in \mathrm{G}^{(\beta)}} \mathcal{D} \mathbf{G} e^{\operatorname{Tr} \mathbf{A G B G}{ }^{*}},
$$

where $\mathrm{G}^{(\beta)}=\mathrm{O}, \mathrm{U}, \mathrm{Sp}$ are respectively the orthogonal/unitary/symplectic $N$-dimensional groups.

From the spectral decomposition of A and $\mathbf{B}$, it is clear that the HCIZ integral only depends on their eigenvalues $\boldsymbol{a}$ and $\boldsymbol{b}$, so that we will denote it by $\mathcal{I}^{(\beta)}(\boldsymbol{a}, \boldsymbol{b})$ in the following. One may also note that since the vector of eigenvalues is unique up to permutation, the HCIZ integral is necessary a symmetric function in each argument $\boldsymbol{a}$ and $\boldsymbol{b}$.

In particular, in the unitary case $(\beta=2)$, Itzykson and Zuber [21] have established the famous formula bearing their names:

$$
\mathcal{I}^{(2)}(\boldsymbol{a}, \boldsymbol{b})=\left(\prod_{i=1}^{N-1} i !\right) \frac{\operatorname{det}\left(e^{a_{i} b_{j}}\right)}{\Delta(\boldsymbol{a}) \Delta(\boldsymbol{b})},
$$

where $\Delta(\boldsymbol{a}):=\prod_{i<j}\left(a_{i}-a_{j}\right)$, is the Vandermonde determinant. The HCIZ integral has applications in problems directly linked to random matrix theory (RMT) such as the study of the sum of invariant ensembles [22-24], the development of large deviation principles [25], the study of the so-called orbital beta processes [26]. It is also linked to the enumeration of Hurwitz numbers in algebraic geometry $[27,28]$ and to quantum ergodic transport ( [29]), to cite a few recent results.

It is then tempting to try to generalize this formula for arbitrary positive $\beta$, just like one can study the eigenvalue distribution of $\beta$ ensembles in RMT for general $\beta$ [1]. There are several possible natural choices to define the HCIZ "integral" ${ }^{2}$ for a generic $\beta>0$ which all lead to the same result: a natural candidate is to see it as the symmetric eigenfunction of the

\footnotetext{
${ }^{1}$ Some authors define the HCIZ integral with a constant $c_{N, \beta}$ in the exponential function that can be absorbed in one of the matrices $\mathbf{A}$ or $\mathbf{B}$.

${ }^{2}$ For values for $\beta$ outside 1, 2 and 4, we lack a Haar integral representation, but we will still call our object of interest the HCIZ "integral".
} 
so-called Calogero-Moser operator normalized to unity whenever $\boldsymbol{a}$ or $\boldsymbol{b}=(0, \ldots, 0)$. One can then show [30] that the HCIZ integral admits the following representation for general $\beta>0$ :

$$
\mathcal{I}^{(\beta)}(\boldsymbol{a}, \boldsymbol{b})=\sum_{k=0}^{\infty} \sum_{|\lambda|=k} d_{\lambda} \mathrm{j}_{\lambda}^{\left(\frac{2}{\beta}\right)}(\boldsymbol{a}) \mathrm{j}_{\lambda}^{\left(\frac{2}{\beta}\right)}(\boldsymbol{b}),
$$

where the second sum is made over all partitions of size $k$ : that is $\lambda=\left(\lambda_{1}, \lambda_{2}, \ldots\right)$ is a sequence of non-increasing integers such that $\sum \lambda_{i}=k, d_{\lambda}:=\prod_{j=1}^{N} \frac{\Gamma\left(\frac{\beta}{2}(N-j+1)\right)}{\Gamma\left(\frac{\beta}{2}(N-j+1)+\lambda_{j}\right)}$ and the $\mathrm{j}_{\lambda}^{\left(\frac{2}{\beta}\right)}(\boldsymbol{a})$ are the so-called "P" Jack polynomials indexed by the partition $\lambda$. The set Jack polynomials is a one-parameter generalization of the set of Schur Polynomials, which corresponds to the case $\beta=2$. At $\beta=1$ (resp. $\beta=4$ ), the Jack polynomials are the real (resp. quaternionic) zonal polynomials. We refer to [31] and [32] for properties concerning these polynomials.

\subsection{The rank-one case}

In this section and in the rest of the article, we fix one matrix to be of rank one, that is we have $\boldsymbol{b}=(t, 0, \ldots, 0)$, and we denote by:

$$
\mathcal{I}_{\boldsymbol{a}}^{(\beta)}(t):=\mathcal{I}^{(\beta)}(\boldsymbol{a},(t, 0, \ldots, 0)),
$$

the corresponding HCIZ integral that we see as a function of $t$ given the vector $\boldsymbol{a}$. The main reason to study this regime is that the large $N$ behavior of the rank one HCIZ integral is very different from the full rank case, which is known to satisfy a complex variational principle [33,34], where analytical results are hard to obtain, except in some specific cases [35]. Specializing to the rank one case will greatly simplify the results obtained for the full rank case. We will first review known and lesser-known formulas in the literature for the rank one HCIZ; namely the power sum representation (9), the differential operator representation (22), the inverse Laplace representation (26), the spherical Dirichlet average representation (31), and the moment generating function representation (36).

\subsubsection{Power sum representation}

We have from [32] the following simplification for the Jack polynomials:

$$
\mathrm{j}_{\lambda}^{\left(\frac{t}{\beta}\right)}(t, 0, \ldots, 0)=\delta_{\lambda, k}\left(\frac{\beta}{2}\right)^{k} \frac{t^{k}}{k !}
$$

where $\delta_{\lambda, k}=1$ if $\lambda=(k, 0, \ldots)$ and 0 otherwise. This greatly simplifies the expansion (6) and we have:

$$
\mathcal{I}_{\boldsymbol{a}}^{(\beta)}(t)=\sum_{k=0}^{\infty} \frac{\Gamma\left(\frac{N \beta}{2}\right)}{\Gamma\left(\frac{N \beta}{2}+k\right)} g_{k}^{\left(\frac{2}{\beta}\right)}(\boldsymbol{a}) t^{k}
$$

where $\mathrm{g}_{k}^{\left(\frac{2}{\beta}\right)}(\boldsymbol{a}):=\frac{1}{k !}\left(\frac{\beta}{2}\right)^{k} \mathrm{j}_{k}^{\left(\frac{2}{\beta}\right)}(\boldsymbol{a})$. These normalized Jack polynomials admit a simple formula for their generating function, which can be taken as their definition:

$$
\prod_{i=1}^{N}\left(1-a_{i} t\right)^{-\frac{\beta}{2}}=\sum_{k=0}^{\infty} g_{k}^{\left(\frac{2}{\beta}\right)}(\boldsymbol{a}) t^{k} .
$$


In particular, we see that if for $m \in \mathbb{N}$, we denote by $\boldsymbol{a}^{\otimes m}=\left(a_{1}, \ldots, a_{1}, \ldots, a_{N}, \ldots, a_{N}\right)$ the vector of size $m N$ obtained by making $m$ copies of the entries of the vector $\boldsymbol{a}$, we have:

$$
\mathrm{g}_{k}^{\left(\frac{2}{\beta}\right)}(\boldsymbol{a})=\mathrm{g}_{k}^{\left(\frac{2 m}{\beta}\right)}\left(\boldsymbol{a}^{\otimes m}\right),
$$

from which we derive the following $\beta \leftrightarrow N$ symmetry satisfied by the rank one HCIZ:

$$
\mathcal{I}_{\boldsymbol{a}}^{(\beta)}(t)=\mathcal{I}_{\boldsymbol{a}^{\otimes m}}^{\left(\frac{\beta}{m}\right)}(t) .
$$

In particular, if $\beta$ is an integer we can always reduce to the $\beta=1$ case since we have:

$$
\mathcal{I}_{\boldsymbol{a}}^{(\beta)}(t)=\mathcal{I}_{\boldsymbol{a}^{\otimes \beta}}^{(1)}(t) \quad \text { (for } \beta \text { integer) }
$$

The normalized Jack polynomial $g_{k}^{\left(\frac{2}{\beta}\right)}$ (.) can be decomposed into the power sum symmetric polynomials which are defined for an integer $k$ by:

$$
\mathrm{p}_{k}(\boldsymbol{a}):=\sum_{i=1}^{N} a_{i}^{k}
$$

that is, they are the unnormalized moments of the discrete measure $\mu_{a}(x)=\frac{1}{N} \sum_{i} \delta\left(x-a_{i}\right)$, where $\delta($.$) is the Dirac mass distribution. They admit the following simple formula for their$ generating function which follows from the power sum expansion of the logarithm:

$$
\log \left(\prod_{i}^{N}\left(1-a_{i} t\right)^{-1}\right)=\sum_{k=1}^{\infty} \frac{t^{k}}{k} \mathrm{p}_{k}(\boldsymbol{a}) .
$$

Combining (10) and (14), we can decompose the $g_{k}^{\left(\frac{2}{\beta}\right)}($.$) in terms of the power sum poly-$ nomials which gives:

$$
g_{k}^{\left(\frac{2}{\beta}\right)}(\boldsymbol{a})=\sum_{1 j_{1}+\cdots+k j_{k}=k}\left(\frac{\beta}{2}\right)^{j_{1}+\cdots+j_{k}} \prod_{i=1}^{k} \frac{\mathrm{p}_{i}(\boldsymbol{a})^{j_{i}}}{i j_{i} j_{i} !} .
$$

The first few terms are given by:

1. $g_{0}^{\left(\frac{2}{\beta}\right)}(\boldsymbol{a})=1$

2. $\mathrm{g}_{1}^{\left(\frac{2}{\beta}\right)}(\boldsymbol{a})=\frac{\beta}{2} \mathrm{p}_{1}(\boldsymbol{a})$

3. $\mathrm{g}_{2}^{\left(\frac{2}{\beta}\right)}(\boldsymbol{a})=\frac{1}{2}\left(\frac{\beta}{2} \mathrm{p}_{2}(\boldsymbol{a})+\left(\frac{\beta}{2} \mathrm{p}_{1}(\boldsymbol{a})\right)^{2}\right)$

4. $\mathrm{g}_{3}^{\left(\frac{2}{\beta}\right)}(\boldsymbol{a})=\frac{1}{3} \frac{\beta}{2} \mathrm{p}_{3}(\boldsymbol{a})+\frac{1}{2}\left(\frac{\beta}{2} \mathrm{p}_{2}(\boldsymbol{a})\right)\left(\frac{\beta}{2} \mathrm{p}_{1}(\boldsymbol{a})\right)+\frac{1}{6}\left(\frac{\beta}{2} \mathrm{p}_{1}(\boldsymbol{a})\right)^{3}$

and we have the recurrence relation:

$$
k g_{k}^{\left(\frac{2}{\beta}\right)}(\boldsymbol{a})=\frac{\beta}{2} \sum_{l=1}^{k} \mathrm{~g}_{k-l}^{\left(\frac{2}{\beta}\right)}(\boldsymbol{a}) \mathrm{p}_{l}(\boldsymbol{a}) .
$$


Remark: Note that in the unitary case, this simplifies to:

$$
\mathcal{I}_{\boldsymbol{a}}^{(2)}(t)=\sum_{k=0}^{\infty}\left(\frac{(N-1) !}{(k+N-1) !} \mathrm{h}_{k}(\boldsymbol{a})\right) t^{k},
$$

where the $\mathrm{h}_{k}(\boldsymbol{a})$ are the complete homogeneous symmetric polynomials:

$$
\mathrm{h}_{k}(\boldsymbol{a}):=\sum_{1 \leq j_{1} \leq \cdots \leq j_{k} \leq N} a_{j_{1}} \ldots a_{j_{k}} .
$$

This power sum expression in the unitary case can actually be derived from the Itzykson-Zuber formula (5) using the Brézin-Hikami trick [36] and the identity:

$$
\mathrm{h}_{k}(\boldsymbol{a})=\left(\sum_{i=1}^{N} \prod_{j \neq j} \frac{a_{i}^{k+N-1}}{a_{j}-a_{i}}\right),
$$

whenever all the $a_{i}$ are distinct.

\subsubsection{Differential operator representation}

We can find a differential operator representation of (9) by first realizing that the coefficient $\frac{\Gamma\left(\frac{N \beta}{2}\right)}{\Gamma\left(\frac{N \beta}{2}+k\right)}$ is precisely the inverse of the coefficient of

$$
(-1)^{k} \frac{\mathrm{d}^{k}}{\mathrm{~d} t^{k}}\left[t^{-\frac{N \beta}{2}}\right]=\frac{\Gamma\left(\frac{N \beta}{2}+k\right)}{\Gamma\left(\frac{N \beta}{2}\right)} t^{-\frac{N \beta}{2}-k} .
$$

If we denote by $U_{a}^{(\beta)}(z)$ the characteristic polynomial raised to the power $-\frac{\beta}{2}$ of the matrix with eigenvalues $\boldsymbol{a}$ :

$$
U_{a}^{(\beta)}(z):=\prod_{i=1}^{N}\left(z-a_{i}\right)^{-\frac{\beta}{2}}=e^{-\frac{N \beta}{2} \int d u \log (z-u) \mu_{a}(u)},
$$

with $\mu_{\boldsymbol{a}}(x)=\frac{1}{N} \sum_{i=1}^{N} \delta\left(x-a_{i}\right)$. By using the formula for the generating function of the Jack polynomials (10), we get the following differential operator form for the HCIZ rank one integral,

$$
U_{a}^{(\beta)}(z)=\mathcal{I}_{a}^{(\beta)}(-\mathrm{D}) z^{-\frac{N \beta}{2}}
$$

where $\mathrm{D}^{k}:=\mathrm{d}^{k} / \mathrm{d} z^{k}$. The term $z^{-\frac{N \beta}{2}}$ can be viewed as $U_{0}^{(\beta)}(z)$ for the null matrix.

Equation (22) could have been taken as an alternative definition for the rank one HCIZ integral for general $\beta>0$. 


\subsubsection{Inverse Laplace representation}

From the power sum relation (9), we can express the HCIZ integral in terms of the inverse Laplace transform $\mathcal{L}_{p}^{-1}[$.$] , using (10), we have for t>0$ :

$$
\begin{aligned}
& \mathcal{I}_{\boldsymbol{a}}^{(\beta)}(t)=\left(\frac{\Gamma\left(\frac{N \beta}{2}\right)}{t^{\frac{N \beta}{2}-1}}\right) \sum_{k=0}^{\infty} g_{k}^{\left(\frac{2}{\beta}\right)}(\boldsymbol{a}) \frac{1}{\Gamma\left(\frac{N \beta}{2}+k\right)} t^{\frac{N \beta}{2}+k-1}, \\
& \mathcal{I}_{\boldsymbol{a}}^{(\beta)}(t)=\left(\frac{\Gamma\left(\frac{N \beta}{2}\right)}{t^{\frac{N \beta}{2}-1}}\right) \sum_{k=0}^{\infty} g_{k}^{\left(\frac{2}{\beta}\right)}(\boldsymbol{a}) \mathcal{L}_{z}^{-1}\left[\frac{1}{z^{\frac{N \beta}{2}+k}}\right](t), \\
& \mathcal{I}_{\boldsymbol{a}}^{(\beta)}(t)=\left(\frac{\Gamma\left(\frac{N \beta}{2}\right)}{t^{\frac{N \beta}{2}-1}}\right) \mathcal{L}_{z}^{-1}\left[\frac{1}{z^{\frac{N \beta}{2}}} \sum_{k=0}^{\infty} g_{k}^{\left(\frac{2}{\beta}\right)}(\boldsymbol{a}) \frac{1}{z^{k}}\right](t) .
\end{aligned}
$$

By applying the generating function formula (10) for $t=\frac{1}{z}$, we get the following Inverse Laplace representation:

$$
\mathcal{I}_{\boldsymbol{a}}^{(\beta)}(t)=\left(\frac{\Gamma\left(\frac{\beta}{2} N\right)}{t^{\frac{\beta}{2} N-1}}\right) \mathcal{L}_{z}^{-1}\left[U_{\boldsymbol{a}}^{(\beta)}(z)\right](t) \quad(t>0),
$$

with $U_{\boldsymbol{a}}^{(\beta)}($.$) defined in (21), which gives in explicit form:$

$$
\mathcal{I}_{\boldsymbol{a}}^{(\beta)}(t)=\left(\frac{\Gamma\left(\frac{\beta}{2} N\right)}{t^{\frac{\beta}{2} N-1}}\right) \frac{1}{2 \pi \mathrm{i}} \int_{\gamma-\mathrm{i} \infty}^{\gamma+\mathrm{i} \infty} d z e^{t z} \prod_{i=1}^{N}\left(z-a_{i}\right)^{-\frac{\beta}{2}} \quad\left(t>0 \text { and } \gamma>a_{\max }\right) .
$$

Remark: For the orthogonal and unitary cases, this formula could have been deduced from the definition of the HCIZ integral, by use of the Gaussian integration, see for example [1], and for the case $\beta>0$ this can be deduce from the spiked $\beta$-Wishart ensemble of [37].

Remark: Note that from this expression, we clearly see the $\beta \leftrightarrow N$ symmetry (12).

\subsubsection{Spherical Dirichlet integral representation}

Another way to generalize the rank one HCIZ integral to arbitrary $\beta$ is to express it as the average of a simple function over some $\beta$ dependent measure. In the classical case $\beta=1,2,4$, from the definition (4), when the matrix $\mathbf{B}$ is a projector of rank one, we can re-express the HCIZ integral as:

$$
\mathcal{I}_{\boldsymbol{a}}^{(\beta)}(t)=\int_{\mathbb{S}_{\beta}^{N-1}} d \boldsymbol{\sigma} e^{t \sum_{i=1}^{N} a_{i}\left(\sum_{b=1}^{\beta} \sigma_{i, b}^{2}\right)} \quad(\beta=1,2,4),
$$

with $\mathbb{S}_{\beta}^{N-1}:=\left\{\boldsymbol{\sigma} \in \mathbb{R}^{N \beta} \mid \sum_{i=1}^{N} \sum_{b=1}^{\beta} \sigma_{i, b}^{2}=1\right\}$, in particular $\mathbb{S}_{1}^{N-1}=\mathbb{S}^{N-1}$ is the usual $N$ dimensional real sphere. We can then make $N$ times the $\beta$ polar change of coordinates $x_{i}^{2}=\sum_{b=1}^{\beta} \sigma_{i, \beta}^{2}$, from which we find:

$$
\mathcal{I}_{\boldsymbol{a}}^{(\beta)}(t) \propto \int_{\mathbb{S}^{N-1}} d \boldsymbol{x}\left|x_{1} \ldots x_{N}\right|^{\beta-1} e^{t \sum_{i=1}^{N} a_{i} x_{i}^{2}} \quad(\beta=1,2,4) .
$$


Following [38], we can generalize the above equation to arbitrary $\beta>0$ by introducing the following $\alpha$-spherical Dirichlet distribution with $\alpha \geq 0$ defined on the real sphere $\mathbb{S}^{N-1}$ as

$$
\mu^{(\alpha)}(x):=\frac{\Gamma\left(\frac{N \alpha}{2}\right)}{\Gamma\left(\frac{\alpha}{2}\right)^{N}}\left|x_{1} \ldots x_{N}\right|^{\alpha-1} .
$$

Using this measure, we could define the rank one HCIZ integral for arbitrary $\beta>0$ by

$$
\mathcal{I}_{\boldsymbol{a}}^{(\beta)}(t)=\mathbb{E}_{\boldsymbol{v} \sim \mu(\beta)}\left[e^{\left(\boldsymbol{v}^{*} \underline{\boldsymbol{a}} \boldsymbol{v}\right) t}\right]
$$

As explained nicely in [38], the parameter $\alpha$ determines how the mass is concentrated on the sphere and we have in particular:

1. $\mu^{(1)}($.$) is the uniform measure on the sphere$

2. $\mu^{(0)}(\boldsymbol{x})=\frac{1}{2 N} \sum_{i=1}^{2 N} \delta\left(\boldsymbol{x} \pm \boldsymbol{e}_{\boldsymbol{i}}\right)$, where $\boldsymbol{e}_{\boldsymbol{i}}$ is the $i^{\text {th }}$ vector of the canonical basis.

3. $\mu^{(\infty)}(x)=\frac{1}{2^{N}} \sum_{i=1}^{2^{N}} \delta\left(x-\frac{1}{\sqrt{N}}( \pm 1, \ldots, \pm 1)\right)$.

This intuitive generalization only works for the rank one case and is therefore less general than our definition (6) using Jack polynomials. It is important to verify that (31) can be derived from our original definition. As noted by the above authors [38], if we denote by $\underline{\boldsymbol{a}}=\operatorname{Diag}(\boldsymbol{a})=\operatorname{Diag}\left(a_{1}, \ldots, a_{N}\right)$, we have:

$$
U_{\boldsymbol{a}}^{(\beta)}(z)=\int_{\mathbb{S}^{N-1}} d x\left(z-v^{*} \underline{a} v\right)^{-\frac{N \beta}{2}} \mu^{(\beta)}(x),
$$

so with the inverse Laplace representation (26), we get:

$$
\mathcal{I}_{\boldsymbol{a}}(t)=\left(\frac{\Gamma\left(\frac{\beta}{2} N\right)}{t^{\frac{\beta}{2} N-1}}\right) \mathcal{L}_{z}^{-1}\left[(z)^{-N \frac{\beta}{2}}\right] \mathbb{E}_{\boldsymbol{v} \sim \mu(\beta)}\left[e^{\left(\boldsymbol{v}^{*} \underline{\boldsymbol{a}} \boldsymbol{v}\right) t}\right],
$$

from which we recover (31).

\subsubsection{Moment generating function representation}

We finish this section with an important formula for the rest of this article. If we now make the following change of variable $\boldsymbol{d}=\left(d_{i}, \ldots, d_{N}\right)$ with $d_{i}=v_{i}^{2}$ in (31), then we have:

$$
\mathcal{I}_{\boldsymbol{a}}^{(\beta)}(t)=\mathbb{E}_{\boldsymbol{d} \sim \mu_{\mathrm{Dir}}}\left[e^{t \boldsymbol{a}^{*} \boldsymbol{d}}\right],
$$

where $\boldsymbol{d}$ follows the (planar) Dirichlet distribution with parameter $\left(\frac{\beta}{2}, \ldots, \frac{\beta}{2}\right)$ : its probability density function is defined over the simplex $\Delta=\left\{x_{i} \in(0,1) \mid \sum x_{i}=1\right\}$ and is given by:

$$
\mu_{\text {Dir }}(\boldsymbol{x})=\frac{1}{C_{\beta, N}}\left|x_{1} \ldots x_{N}\right|^{\frac{\beta}{2}-1}
$$

then doing the change of variable $X=\boldsymbol{a}^{*} \boldsymbol{d}$ allows us to represent the HCIZ integral as a moment generating function: 


$$
\mathcal{I}_{a}^{(\beta)}(t)=\mathbb{E}_{X \sim \mathcal{M}_{\frac{N \beta}{2}, a}}\left[e^{t X}\right]
$$

The distribution $\mathcal{M}_{\frac{N \beta}{2}, a}$ is known as a mean Dirichlet process or as the (discrete) Markov-Krein transform (MKT) of the vector $\boldsymbol{a}$, with parameter $\frac{N \beta}{2}$. One should think of the transformed variable $X$ as a random convex combination of the $a_{i}$ 's, its support is naturally given by the extreme values of $\boldsymbol{a}$ namely $\left[a_{\min }, a_{\max }\right]$, with $a_{\min }:=\min \boldsymbol{a}$ and $a_{\max }:=\max \boldsymbol{a}$. By the symmetry of the Dirichlet process the first moment is preserved: $\mathbb{E}[X]=\frac{1}{N} \sum_{i=1}^{N} a_{i}$. and for $t>0$, we see that the bounds:

$$
e^{t a_{\min }} \leq \mathcal{I}_{\boldsymbol{a}}^{(\beta)}(t) \leq e^{t a_{\max }} \quad(t>0),
$$

are preserved. Those are immediate for $\beta=1,2,4$ from the definition of the HCIZ integral, Next we give a formula that we will prove later in a more general context, relating the vector $\boldsymbol{a}$ to the distribution $\mathcal{M}_{\frac{N \beta}{2}, \boldsymbol{a}}$ :

$$
\int_{a_{\min }}^{a_{\max }} d x(z-x)^{-\frac{N \beta}{2}} \mathcal{M}_{\frac{N \beta}{2}, a}(x)=U_{a}^{(\beta)}(z) .
$$

\subsection{Large $N$ behavior of the rank one HCIZ}

\subsection{1 $\beta>0$ and relation with free probability}

As explained in the introduction of this section, the main reason to study the rank one HCIZ integral is its large $N$ behavior. In particular, it is known for the three classical value $\beta=1,2,4$ that if we denote by $\boldsymbol{\gamma}=\operatorname{Eigen}\left(\underline{\boldsymbol{a}}+\mathbf{G}^{\prime} \underline{\boldsymbol{b}} \underline{\mathbf{G}}^{\prime *}\right)$, with $\underline{\boldsymbol{a}}=\operatorname{Diag}(\boldsymbol{a})=\operatorname{Diag}\left(a_{1}, \ldots, a_{N}\right)$ and similarly for $\underline{b}$, we have, from the property of the Haar measure, the following formula for the rank one HCIZ:

$$
\mathbb{E}_{\mathbf{G}^{\prime} \in \mathrm{G}^{(\beta)}}\left[\mathcal{I}_{\gamma}^{(\beta)}(t)\right]=\mathcal{I}_{\boldsymbol{a}}^{(\beta)}(t) \mathcal{I}_{\boldsymbol{b}}^{(\beta)}(t) \quad(\beta=1,2,4) .
$$

In the large $N$ limit, we assume that the spectral measure $\mu_{\boldsymbol{a}}(x):=\frac{1}{N} \sum_{i=1}^{N} \delta\left(x-a_{i}\right)$ converges $^{3}$ to a compactly supported deterministic measure $\mu_{A}$ such that $\min \boldsymbol{a} \rightarrow a_{\min }$ and $\max \boldsymbol{a} \rightarrow a_{\max }$, where $a_{\min }$ and $a_{\max }$ are the left and right extremities of the support of the measure $\mu_{A}$.

We expect to have some self-averaging in the LHS of (39), so that we can remove the expectation, making the logarithm of the HCIZ additive for the free convolution and therefore directly connected to the famous $\mathcal{R}$-transform of RMT. To establish such relation, we perform a standard saddle point analysis in (27) for $\beta>0$ :

$$
\begin{aligned}
& \mathcal{I}_{\boldsymbol{a}}^{(\beta)}(N t)=\left(\frac{\Gamma\left(\frac{\beta}{2} N\right)}{(N t)^{\frac{\beta}{2} N-1}}\right) \frac{1}{2 \pi \mathrm{i}} \int_{\gamma-\mathrm{i} \infty}^{\gamma+\mathrm{i} \infty} d z e^{N t z-\frac{\beta}{2} \sum_{i=1}^{N} \log \left(z-a_{i}\right)}, \\
& \mathcal{I}_{\boldsymbol{a}}^{(\beta)}(N t)=\frac{1}{2 \pi \mathrm{i}} \int_{\gamma-\mathrm{i} \infty}^{\gamma+\mathrm{i} \infty} d z e^{N \mathcal{H}^{(\beta)}(z, t)},
\end{aligned}
$$

with:

\footnotetext{
${ }^{3}$ For simplicity we write $\boldsymbol{a}$ instead of $\boldsymbol{a}_{(N)}$ even though the vector $\boldsymbol{a}$ is $N$-dependent.
} 


$$
\begin{aligned}
\mathcal{H}^{(\beta)}(z, t)= & t z-\frac{\beta}{2} \int d x \log (z-x) \mu_{\boldsymbol{a}}(x)-\frac{\beta}{2} \log (t) \\
& +\frac{1}{N}\left(\log \left(\Gamma\left(\frac{\beta}{2} N\right)\right)-\left(\frac{\beta}{2} N-1\right) \log (N)\right)+\frac{1}{N} \log (t),
\end{aligned}
$$

Now we have by Stirling formula that:

$$
\lim _{N \rightarrow \infty} \frac{1}{N}\left(\log \left(\Gamma\left(\frac{\beta}{2} N\right)\right)-\left(\frac{\beta}{2} N-1\right) \log N\right)+\frac{1}{N} \log z=\frac{\beta}{2} \log \frac{\beta}{2}-\frac{\beta}{2},
$$

and since we have $\mu_{\boldsymbol{a}} \rightarrow \mu_{A}$ we have:

$$
\lim _{N \rightarrow \infty} \frac{1}{N} \log \mathcal{I}_{a}^{(\beta)}(N t)=\mathcal{H}^{(\beta)}\left(z^{*}(t), t\right)
$$

with

$$
\mathcal{H}^{(\beta)}(z, t) \simeq z t-\frac{\beta}{2} \int d x \log (z-x) \mu_{\boldsymbol{a}}(x)-\frac{\beta}{2} \log (t)+\frac{\beta}{2} \log \frac{\beta}{2}-\frac{\beta}{2},
$$

and $z^{*}(t)$ solution of :

$$
\left\{\begin{array}{l}
\partial_{z} \mathcal{H}^{(\beta)}(z, t)=0 \\
t-\frac{\beta}{2} \mathcal{G}_{\mu_{A}}(z)=0
\end{array},\right.
$$

where $\mathcal{G}_{\mu}($.$) is defined in (2). That is z^{*}(t)=\mathcal{G}_{\mu_{\mathcal{A}}}{ }^{(-1)}\left(\frac{2}{\beta} t\right)$ for $t$ close enough to the origin. One may notice then:

$$
\begin{aligned}
\frac{d}{d z} \mathcal{H}^{(\beta)}\left(z^{*}(t), t\right) & =\mathcal{G}_{\mu_{A}}^{(-1)}\left(\frac{2}{\beta} t\right)+\frac{2}{\beta} t \frac{d}{d t} \mathcal{G}_{\mu_{A}}^{(-1)}\left(\frac{2}{\beta} t\right)-\left(\frac{2}{\beta} t\right) \frac{d}{d t} \mathcal{G}_{\mu_{A}}^{(-1)}\left(\frac{2}{\beta} t\right)-\frac{\beta}{2} \frac{1}{t}, \\
\frac{d}{d t} \mathcal{H}^{(\beta)}\left(z^{*}(t), t\right) & =\mathcal{G}_{\mu_{A}}^{(-1)}\left(\frac{2}{\beta} t\right)-\frac{\beta}{2} \frac{1}{t},
\end{aligned}
$$

so that at the end we have the following simple formula:

$$
\lim _{N \rightarrow \infty} \frac{1}{N} \frac{d}{d t} \log \mathcal{I}_{\boldsymbol{a}}^{(\beta)}(N t)=\mathcal{R}_{\mu_{A}}\left(\frac{2}{\beta} t\right)(t \text { close to } 0),
$$

where $\mathcal{R}_{\mu_{A}}($.$) is defined in (1). This result was first derived for \beta=1,2$ by Parisi [39] and made rigorous by Guionnet and Maïda [40] for $\beta=1,2$ using Gaussian concentration and under a more general setting. In particular, the asymptotic for all $t$ and not just close to origin is derived and one can see that there is a phase transition at a certain $t^{*}$ above which the asymptotic (48) is no more true, we refer to [40] for more details.

Remark: Note that for integer $\beta$, this formula is consistent with the $\beta \leftrightarrow N$ symmetry (12) since $\mu_{\boldsymbol{a}^{\otimes \beta}} \rightarrow \mu_{A}$. 


\subsubsection{Infinite temperature regime $(\beta \rightarrow 0)$ and classical convolution}

In the previous subsection, the parameter $\beta$ was restricted to real positive values. The aim of this section is to describe the extreme value zero. To get the behavior for this value, we will use equivalently the limiting behavior of the Jack polynomials and the Dirichlet average representation (31).

By the recurrence relation (16) satisfied by the $g_{k}($.$) and using properties of the gamma$ function, we have for $k>0$ :

- $\lim _{\beta \rightarrow 0}\left(\frac{2}{\beta}\right) g_{k}^{\left(\frac{2}{\beta}\right)}(\boldsymbol{a})=\frac{\operatorname{Tr} \underline{\underline{a}}^{k}}{k}$, where we recall $\underline{\boldsymbol{a}}:=\operatorname{Diag}(\boldsymbol{a})$.

- $\lim _{\beta \rightarrow 0} \frac{\Gamma\left(\frac{\beta}{2} N\right)\left(\frac{\beta}{2}\right)}{\Gamma\left(\frac{\beta}{2} N+k\right)}=\frac{1}{N(k-1) !}$,

so that in the end we get for the HCIZ integral:

$$
\lim _{\beta \rightarrow 0} \mathcal{I}_{\boldsymbol{a}}^{(\beta)}(t)=\sum_{k=0}^{\infty} \frac{m_{k}(\boldsymbol{a})}{k !} t^{k},
$$

with $m_{k}(\boldsymbol{a}):=\frac{1}{N} \sum_{k=1}^{N} a_{i}^{k}$, is the $k^{t h}$ moment of the (random) distribution $\mu_{\boldsymbol{a}}$.

This is also consistent will the Dirichlet average representation, since in this case the measure degenerates at the poles $\pm \boldsymbol{e}_{i}$ with $\boldsymbol{e}_{i}$ the $i^{\text {th }}$ canonical vector.

$$
\begin{aligned}
& \frac{1}{2 N} \sum \delta\left(\boldsymbol{v} \pm \boldsymbol{e}_{i}\right) e^{\sum_{j=1}^{N} a_{j} v_{j}^{2} t}=\frac{1}{N} \sum_{i=1}^{N} e^{a_{i} t}, \\
& \frac{1}{2 N} \sum \delta\left(\boldsymbol{v} \pm \boldsymbol{e}_{i}\right) e^{\sum_{i=1}^{N} a_{j} v_{j}^{2} t}=\sum_{k=0}^{\infty} \frac{m_{k}(\boldsymbol{a})}{k !} t^{k},
\end{aligned}
$$

that is we have:

$$
\lim _{\beta \searrow 0} \mathcal{I}_{\boldsymbol{a}}^{(\beta)}(t)=\mathbb{E}_{X \sim \mu_{A}}\left[e^{t X}\right] .
$$

In other words, in the $\beta$ goes to zero limit, the rank one HCIZ is nothing else than the classical generating function of the moments and under the same assumptions as in Section 2.3.1, this property is preserved by the limit $N \rightarrow \infty$. In the Markov-Krein language, the variable $X$ can only take values $a_{i}$ each with probability $1 / N$ hence its measure is equal to the discrete measure $\mu_{\boldsymbol{a}}$. In particular, the logarithm of the rank-one HCIZ is the generating function of the classical cumulants. This is expected since, in the theory of $\beta$-ensembles, the parameter $\beta$ measures the strength of the interactions between the eigenvalues, at $\beta=0$ there is no interaction and one recovers classical objects. It is worth noting that if we denote by $\mathbf{P}$ a $N \times N$ permutation matrix, we can express the rank one HCIZ at $\beta=0$ as a Haar integral:

$$
\mathcal{I}_{\boldsymbol{a}}^{(0)}(t)=\int_{\mathbf{P} \in \operatorname{Sym}(N)} \mathcal{D} \mathbf{P} e^{t\left(\underline{P}^{\underline{a}} \mathbf{P}^{*}\right)_{11}},
$$

where $\mathcal{D} \mathbf{P}$ is the normalized (discrete) counting measure of the permutation group. This is actually a special case of the formula of the full-rank case, since we have:

$$
\mathcal{I}^{(0)}(\boldsymbol{a}, \boldsymbol{b})=\int_{\mathbf{P} \in \operatorname{Sym}(N)} \mathcal{D P} e^{\left(\mathrm{P} \underline{\boldsymbol{a}} \underline{\mathrm{P}}^{*} \underline{\boldsymbol{b}}\right)}
$$


Remark: Similarly in the freezing regime $(\beta \rightarrow \infty)$ we get:

$$
\lim _{\beta \rightarrow \infty} \mathcal{I}_{\boldsymbol{a}}^{(\beta)}(t)=e^{\left(\frac{1}{N} \operatorname{Tr} \underline{\boldsymbol{a}}\right) t}
$$

In this limit and with this scaling, the HCIZ integral only captures the mean of the limiting distribution. As a consequence, it does not provide much information on the complex structure of this regime where one expects the eigenvalues to "freeze" on a lattice, see for example [41].

\section{Negative $\beta$ and finite free convolution}

\subsection{Definition}

It is tempting to generalize the HCIZ formula to negative value $\beta=-\gamma, \gamma>0$. To do so, let's introduce the following generalization of the Jack polynomials:

$$
\prod_{i=1}^{N}\left(1-a_{i} t\right)^{\frac{\gamma}{2}}:=\sum_{k=0}^{\infty} g_{k}^{\left(-\frac{2}{\gamma}\right)}(\boldsymbol{a}) t^{k} .
$$

Remark: If $\gamma$ is even $(\gamma \in 2 \mathbb{N})$, then we have $g_{k}^{\left(-\frac{2}{\gamma}\right)}(\boldsymbol{a})=0$ for $k>\frac{N \gamma}{2}$, since the LHS is a polynomial in $t$. In particular, we have for $\gamma=2$ :

$$
g_{k}^{(-1)}(\boldsymbol{a})=\left\{\begin{array}{ll}
(-1)^{k} \mathrm{e}_{k}(\boldsymbol{a}) & \text { for } k \leq N \\
0 & \text { otherwise }
\end{array},\right.
$$

where the $\mathrm{e}_{k}($.$) are the elementary symmetric polynomials:$

$$
\mathrm{e}_{k}(\boldsymbol{a}):=\sum_{1 \leq j_{1}<\cdots<j_{k} \leq N} a_{j_{1}} \ldots a_{j_{k}} .
$$

By Euler's formula:

$$
\Gamma(1-t)=\frac{\pi}{\Gamma(t) \sin \pi t} \quad \text { for } t \in \mathbb{C} \backslash \mathbb{N},
$$

we can then formally define the rank one HCIZ integral for negative $\beta=-\gamma$ by simply taking (59) with the definition of the negative Jack polynomials (56) in (9). By singularity of the gamma function at negative integers, this extension of the definition of the HCIZ integral to negative values is, at $N$ fixed, only true for specific values of the parameter $\gamma$ due to the term:

$$
\frac{\Gamma\left(\frac{N \gamma}{2}-k+1\right)}{\Gamma\left(\frac{N \gamma}{2}+1\right)} g_{k}^{\left(-\frac{2}{\gamma}\right)}(\boldsymbol{a}),
$$

in the sum. For $\gamma$ even, thanks to the previous remark, we see that there is no problem since we can fix it to be equal to zero for $k>\frac{N \gamma}{2}$ and hence there is no singularity. So if we define by:

$$
R_{(N)}:=\left\{\gamma \in \mathbb{R}_{+} \text {such that } \frac{N \gamma}{2} \notin \mathbb{N} \text { or } \gamma \in 2 \mathbb{N}\right\}
$$


the set of admissible values of $\gamma$, then we can define the HCIZ at negative values by:

$$
\mathcal{I}_{\boldsymbol{a}}^{(-\gamma)}(-t):=\sum_{k=0}^{\infty} \frac{\Gamma\left(\frac{N \gamma}{2}-k+1\right)}{\Gamma\left(\frac{N \gamma}{2}+1\right)} \mathrm{g}_{k}^{\left(-\frac{2}{\gamma}\right)}(\boldsymbol{a}) t^{k} \quad\left(\text { for } \gamma \in R_{(N)}\right) .
$$

Similarly to the positive case, we have:

$$
\mathrm{D}^{k} t^{\frac{N \gamma}{2}}=\frac{\Gamma\left(\frac{N \gamma}{2}+1\right)}{\Gamma\left(\frac{N \gamma}{2}-k+1\right)} t^{\frac{N \gamma}{2}-k}
$$

which leads us to the following differential operator representation:

$$
\prod_{i=1}^{N}\left(z-a_{i}\right)^{\frac{\gamma}{2}}=\mathcal{I}_{\boldsymbol{a}}^{\left(-\frac{2}{\gamma}\right)}(-\mathrm{D}) z^{\frac{N \gamma}{2}} \quad\left(\text { for } \gamma \in R_{(N)}\right)
$$

Again for $\frac{N \gamma}{2} \in \mathbb{N}$, the RHS of (64) is a sum of derivatives of a polynomial, hence a polynomial, whereas the LHS (for $\gamma \notin 2 \mathbb{N}$ ) is a formal power sum and, therefore, strict equality is not possible. When $\gamma \in 2 \mathbb{N}$, we have an equality between two polynomials.

Remark: By the limits:

- $\lim _{\gamma \rightarrow 0} \frac{2}{\gamma} g_{k}^{\left(-\frac{2}{\gamma}\right)}(\boldsymbol{a})=-\frac{\operatorname{Tr} \underline{\underline{a}}^{k}}{k}$

- $\lim _{\gamma \rightarrow 0} \Gamma\left(\frac{\gamma N}{2}\right) \frac{\gamma}{2}=\frac{1}{N(k-1) !}$

we see that we have $\mathcal{I}_{\boldsymbol{a}}^{\left(0^{-}\right)}(-t)=\mathcal{I}_{\boldsymbol{a}}^{\left(0^{+}\right)}(t)=\sum_{k=0}^{\infty} \frac{m_{k}(\boldsymbol{a})}{k !} t^{k}$.

\subsection{The special case $\gamma$ even}

In the rest of this section, we look at the special case $\gamma \in 2 \mathbb{N}=\{2,4,6, \ldots\}$. It is immediate from the definition of the negative Jack polynomials that we have again a $\gamma \leftrightarrow N$ symmetry, in particular, for $m \in \mathbb{N}, k<\frac{N m \gamma}{2}$, using (57) we have:

$$
g_{k}^{\left(-\frac{2}{m \gamma}\right)}(\boldsymbol{a})=(-1)^{k} \mathrm{e}_{k}\left(\boldsymbol{a}^{\otimes m}\right),
$$

so we can specialize to the case $\gamma=2$ without any loss of generality. In this setting we have:

$$
\begin{aligned}
& \mathcal{I}_{\boldsymbol{a}}^{(-2)}(-t)=\sum_{k=0}^{N} \frac{(N-k) !}{N !}(-1)^{k} \mathrm{e}_{k}(\boldsymbol{a}) t^{k} \\
& \mathcal{I}_{\boldsymbol{a}}^{(-2)}(-t)=\left(\frac{t^{N+1}}{N !}\right) \sum_{k=0}^{N}(N-k) !(-1)^{k} \mathrm{e}_{k}(\boldsymbol{a}) t^{k-N-1},
\end{aligned}
$$

but since we have:

$$
(N-k) ! t^{k-N-1}=\mathcal{L}_{z}\left[z^{N-k}\right](t),
$$

where $\mathcal{L}_{z}[$.$] is the Laplace transform with respect to the variable z$, we get: 


$$
\begin{aligned}
& \mathcal{I}_{\boldsymbol{a}}^{(-2)}(-t)=\left(\frac{t^{N+1}}{N !}\right) \mathcal{L}_{z}\left[\sum_{k=0}^{N}(-1)^{k} \mathrm{e}_{k}(\boldsymbol{a}) z^{N-k}\right], \\
& \mathcal{I}_{\boldsymbol{a}}^{(-2)}(-t)=\left(\frac{t^{N+1}}{N !}\right) \mathcal{L}_{z}\left[\prod_{i=1}^{N}\left(z-a_{i}\right)\right], \\
& \mathcal{I}_{\boldsymbol{a}}^{(-2)}(-t)=\left(\frac{t^{N+1}}{N !}\right) \int_{0}^{\infty} d z e^{-z t+\int d u \log (z-u) \mu_{\boldsymbol{a}}(u)} .
\end{aligned}
$$

This expression is the negative counterpart of (26). From (70), it is clear that the large $N$ asymptotics of the integral is dominated by the same saddle point as the one in Section 2.3.1, so under the same assumptions as in Section 2.3.1, we directly obtain the following asymptotics:

$$
\lim _{N \rightarrow \infty}-\frac{1}{N} \frac{d}{d t} \log \mathcal{I}_{\boldsymbol{a}}^{(-2)}(-N t)=\mathcal{R}_{\mu_{A}}(t) .
$$

The case for general $\gamma \in 2 \mathbb{N}$ follow easily using the $\gamma \leftrightarrow N$ symmetry (65).

\subsection{Link with finite free convolution}

In [19] and [18] the authors have introduced the following convolution, known as the finite free convolution: Let $\mu_{\boldsymbol{a}}(x)=\frac{1}{N} \sum_{i=1}^{N} \delta\left(x-a_{i}\right)$ and $\mu_{\boldsymbol{b}}(x)=\frac{1}{N} \sum_{i=1}^{N} \delta\left(x-b_{i}\right)$ be two finite distributions of the same size $N$. Then, since we are at $\gamma=2$, (64) simply becomes:

$$
\prod_{i=1}^{N}\left(t-a_{i}\right)=\mathcal{I}_{\boldsymbol{a}}^{(-2)}(-\mathrm{D}) t^{N},
$$

and similarly for $\boldsymbol{b}$. Their finite free convolution denoted by:

$$
\mu_{c}=\mu_{a} \boxplus_{N} \mu_{b}
$$

is then defined as the unique, well behaved, finite $N$ probability measure on the (real) points $c_{i}$ which are solutions of:

$$
\prod_{i=1}^{N}\left(t-c_{i}\right)=\mathcal{I}_{\boldsymbol{a}}^{(-2)}(-\mathrm{D}) \mathcal{I}_{\boldsymbol{b}}^{(-2)}(-\mathrm{D}) t^{N} .
$$

We refer to [19] and [18] for several other formulations and properties of this convolution. In particular (75) can be restated as:

$$
\mathcal{I}_{\boldsymbol{c}}^{(-2)}(t)=\mathcal{I}_{\boldsymbol{a}}^{(-2)}(t) \mathcal{I}_{\boldsymbol{b}}^{(-2)}(t) \quad \bmod t^{N+1},
$$

where mod $t^{N+1}$ means equality of the power series up to the $N^{\text {th }}$ term, which is obviously needed since we known that $\mathcal{I}_{c}^{(-2)}($.$) is a polynomial of order N$ while the product in the right hand side (RHS) of (76) is a polynomial of order $2 N$. Note that the terms of order higher than $N$ do not contribute in (75). Now, under the same assumptions as in Section 2.3.1, taking the limit $N$ goes to infinity in (76), we can formally remove the mod $t^{N+1}$, so that together with the limit (72), we have:

$$
\lim _{N \rightarrow \infty}\left(\mu_{\boldsymbol{a}} \boxplus_{N} \mu_{\boldsymbol{b}}\right)=\mu_{A} \boxplus \mu_{B},
$$

hence the name finite free convolution. 
Remark: One can define finite free cumulants for this finite free convolution similarly to the way classical and free cumulants are defined in classical and free probability. We refer to [42] for more details. We conclude this section with another interesting point of view, detailed in [18], concerning the finite free convolution that will have a clear analogous in our construction of the $c$-convolution of Section 5. To each finite $N$ measure $\mu_{\boldsymbol{a}}$ we can associate a finite $N$ complex valued measure $\mu_{s}(z)=\frac{1}{N} \sum_{i=1}^{N} \delta\left(z-s_{i}\right)$ that we call the negative MarkovKrein transform ${ }^{4}$ of $\mu_{\boldsymbol{a}}$ such that we have:

$$
\int_{\mathbb{C}} d u(z-u)^{N} \mu_{\boldsymbol{s}}(u)=\prod_{i=1}^{N}\left(z-a_{i}\right),
$$

then plugging (78) in (70), one arrives at (see [18]):

$$
\mathcal{I}_{\boldsymbol{a}}^{(-2)}(t)=\int_{\mathbb{C}} d u e^{t u} \mu_{\boldsymbol{s}}(u) \quad \bmod t^{N+1} .
$$

We note the clear correspondence between the $\beta>0$ case and the $\beta=-2$, in particular we see that (78) is the negative counterpart of (38) at $\beta=-2$ while (79) is the negative counterpart of (36), we see that due to the lack of a Dirichlet representation, the negative Markov-Krein transform is complex valued. Nevertheless, (79) together with (76) indicates that the finite free convolution can be understood - up to a truncation operation - as a convolution of the negative Markov-Krein transforms.

\section{HCIZ at the high temperature limit $\frac{N \beta}{2} \rightarrow c$}

\subsection{Definition and notations}

From Section 2.3, we have seen that the HCIZ integral exhibits a drastic change of behavior as $\beta$ gets close to zero. As is standard statistical physics (see for example [43] for a model linked to RMT), to introduce a continuous phase transition between the two regimes, we take $\beta$ going slowly to 0 by which we mean $\frac{N \beta}{2} \rightarrow c$, where $c \geq 0$ is a tunable parameter ${ }^{5}$. Since this limit only makes sense as $N$ goes to infinity, the goal of this subsection is to make precise what we mean by HCIZ at high temperature and show that most of the representations of Section 2.2 admit a high-temperature counterpart. Let's fix a measure $\mu_{A}$ with compact support $I$. The corresponding $\mathrm{c}$-HCIZ is defined by:

$$
\mathcal{I}_{\mu}^{[c]}(t):=\sum_{k=0}^{\infty} \frac{\Gamma(c)}{\Gamma(c+k)} \mathrm{g}_{k}^{[c]}(\mu) t^{k},
$$

where the $g_{k}^{[c]}(\mu)$ are defined by taking the $\frac{N \beta}{2} \rightarrow c$ limit in the power sum expansion of the normalized Jack polynomials (15) which gives:

$$
g_{k}^{[c]}(\mu):=\sum_{1 j_{1}+\cdots+k j_{k}=k} c^{j_{1}+\cdots+j_{k}} \prod_{i=1}^{k} \frac{m_{i}(\mu)^{j_{i}}}{i j_{i} j_{i} !},
$$

\footnotetext{
${ }^{4}$ In [18], the distribution $\mu_{\boldsymbol{s}}$ is called the $U$-transform of the set $\boldsymbol{a}$.

${ }^{5}$ Note that even though other scalings could have been chosen, this particular one has already been studied in the RMT literature in a completely different context $[12,14]$, and was shown to exhibit non-trivial limiting objects.
} 
where $m_{i}(\mu)$ is the $i^{\text {th }}$ moment of the measure $\mu$. They satisfy the recurrence:

$$
k \mathrm{~g}_{k}^{[c]}(\mu)=c \sum_{l=1}^{k} \mathrm{~g}_{k-l}^{[c]}(\mu) m_{l}(\mu) .
$$

We define the high temperature analog of $U_{a}^{(\beta)}(z):=\operatorname{det}(z-\underline{a})^{-\beta / 2}$,

$$
U_{\mu}^{[c]}(z):=\exp \left\{-c \int_{I} d x \log (z-x) \mu(x)\right\},
$$

which, by the properties of the logarithm, is analytic for all $\mathbb{C} \backslash\left(-\infty, a_{\max }\right)^{6}$. It can be equivalently represented as:

$$
U_{\mu}^{[c]}(z)=\frac{1}{z^{c}} \sum_{k=0}^{\infty} g_{k}^{[c]}(\mu) \frac{1}{z^{k}},
$$

and is linked to the Stieltjes transform by:

$$
\mathcal{G}_{\mu}(z)=-\frac{1}{c} \frac{d}{d z} \log U_{\mu}^{[c]}(z) .
$$

Using (102) in Section 4.2, one may recover the original distribution thanks to the inversion formula:

$$
\mu(x)=-\frac{1}{c \pi} \frac{d}{d x} \lim _{\eta \searrow 0} \mathfrak{I m} \log \left\{U_{\mu}^{[c]}(x-\mathrm{i} \eta)\right\}
$$

where the derivative has to be understood in the sense of distributions. Next, by doing the same derivation as in Section 2.2.3, we get the following high temperature counterpart of (26):

$$
\mathcal{I}_{\mu}^{[c]}(t)=\frac{\Gamma(c)}{t^{c-1}} \mathcal{L}_{z}^{-1}\left[U_{\mu}^{[c]}(z)\right](t) \quad(t>0),
$$

which can be inverted into:

$$
U_{\mu}^{[c]}(z)=\frac{1}{\Gamma(c)} \mathcal{L}_{t}\left[t^{c-1} \mathcal{I}_{\mu}^{[c]}(t)\right](z) \quad\left(\Re \mathfrak{R e z}>a_{\max }\right),
$$

and then extended analytically to all $z \in \mathbb{C} \backslash\left(-\infty, a_{\max }\right)$. We emphasize that we have assumed the measure $\mu$ to be compactly supported so the complex integral contour in the inverse Laplace transform of (87) can always be deformed to have the branch cut on the left side of the integral contour and hence (87) is well-defined. If we consider a measure $\mu$ with unbounded support (from bellow), the inverse Laplace transform is not necessarily well-defined and equation (87) only makes sense as an equality between formal series. In some cases, we can use a trick similar to a Wick rotation, namely, multiply the argument $z$ by a constant using scaling properties implied by (81) and (83):

$$
\mathcal{I}_{\mu}^{[c]}(t) \rightarrow \mathcal{I}_{\mu}^{[c]}(K t) \Rightarrow U_{\mu}^{[c]}(z) \rightarrow K^{-c} U_{\mu}^{[c]}\left(\frac{z}{K}\right) .
$$

\footnotetext{
${ }^{6}$ One may notice that crossing the branch cut at a point $x_{0}<a_{\min }$ introduces a phase $e^{2 \mathrm{i} \pi c}$, so that when $c$ is an integer one can extend analytically the function to $\mathbb{C} \backslash I$.
} 
If by such a scaling the formal power series now converge, the rescaled functions are then equal on their domain of convergence. In particular, if we look at a measure whose support is of the type $(a, \infty)$, then taking $K=-1$ is the same as considering the measure $\mu(-$.) whose support is $(-\infty,-a)$ which makes the inverse Laplace transform converges. This is reminiscent of the fact that for measures on $\mathbb{R}_{+}$, the Laplace transform is more appropriate analytically than the moment generating function. Following the derivation of Section 2.2.2 together with (84), we have again:

$$
U_{\mu}^{[c]}(z)=\mathcal{I}_{\mu}^{[c]}(-\mathrm{D}) z^{-c}
$$

To establish the high temperature counterpart of (36), one can first fix $\beta_{(N)}=\frac{2 c}{N}$ and a corresponding sequence of finite measures $\mu_{\boldsymbol{a}_{(N)}}$ such that $\mu_{\boldsymbol{a}_{(N)}} \rightarrow \mu$ and $a_{\min (N)}$ and $a_{\max (N)}$ converge towards the edges of the support $I$, and then we take the limit $N \rightarrow \infty$ in (36) accordingly, so that we have:

$$
\mathcal{I}_{\mu}^{[c]}(t)=\mathbb{E}_{X \sim \mathcal{M}_{c, \mu}}\left[e^{t X}\right],
$$

where the measure $\mathcal{M}_{c, \mu}$ is known as the Markov-Krein Transform (MKT) of $\mu$. The MKT is discussed in great details in [44], where the link with RMT is made. The link between the MKT and the HCIZ integral can be derived from results of [45], although the authors do not make this link explicitly and they do not study the high temperature regime.

\subsection{Generalized Stieltjes transform and fractional calculus}

The purpose of this section is to introduce the generalized Stieltjes transform which will improve our understanding of the properties of the MKT. For a measure $v$ with compact support $J$ with left and right extremities $b_{\min }$ and $b_{\max }$, and $s>0$, the generalized Stieltjes transform of order $s$ is defined for all $z \in \mathbb{C} \backslash\left(-\infty, b_{\max }\right)^{7}$ by:

$$
\mathcal{G}_{v}^{(s)}(z):=\int_{J} d x \frac{v(x)}{(z-x)^{s}} .
$$

For $s=1$, we drop the superscript and simply write $\mathcal{G}_{\nu}($.$) as one recovers the usual defini-$ tion of the Stieltjes transform. Taking the Taylor expansion of the power function, one arrives at the following formal expansion for the generalized Stieltjes transform:

$$
\mathcal{G}_{v}^{(s)}(z)=\frac{1}{\Gamma(s)} \sum_{k=0}^{\infty} \frac{\Gamma(s+k)}{k !} m_{k}(v) z^{-k-s}
$$

where $m_{k}(v)$ is the $k^{t h}$ moment of the measure $v$, with the usual convention $m_{0}(v)=1$. It is worth noting that using:

$$
\frac{1}{(z-x)^{s}}=\frac{1}{\Gamma(s)} \int_{0}^{\infty} d t t^{s-1} e^{-t(z-x)}
$$

we can rewrite:

\footnotetext{
${ }^{7}$ For $s$ integer, one can extend the function to $\mathbb{C} \backslash J$.
} 


$$
\mathcal{G}_{v}^{(s)}(z):=\frac{1}{\Gamma(s)} \mathcal{L}_{t}\left[t^{s-1} \mathbb{E}_{X \sim v}\left[e^{t X}\right]\right](z) .
$$

For a measure defined on $\mathbb{R}_{+}$and $s=1$, we recover the fact that up to a sign, the Stieltjes transform is an iterated Laplace transform:

$$
-\mathcal{G}_{v}(-z)=\mathcal{L}_{t}\left[\mathcal{L}_{x}[v(x)](t)\right](z) .
$$

To connect the generalized and standard Stieltjes transforms, observe that for $s>1$, one has:

$$
x^{-s}=\frac{1}{\Gamma(s)} \mathrm{D}^{s-1} x^{-1},
$$

where for $0<s<1, \mathrm{D}^{s-1}=\mathrm{D}^{-(1-s)}$ is the fractional anti-derivative ${ }^{8}$ of order $\alpha=1-s$, defined by:

$$
\mathrm{D}^{-\alpha} f(x):=\frac{1}{\Gamma(\alpha)} \int_{x}^{\infty} d y(y-x)^{\alpha-1} f(y),
$$

and for $s>1$, it is the fractional derivative of order $\alpha=s-1$ :

$$
\mathrm{D}^{\alpha} f(x):=\mathrm{D}^{\alpha-\lfloor\alpha\rfloor} \mathrm{D}^{\lfloor\alpha\rfloor} f(x):=-\frac{1}{\Gamma(\lfloor\alpha\rfloor+1-\alpha)} \int_{x}^{\infty} d y(y-x)^{-\alpha} \frac{d^{\lfloor\alpha\rfloor+1}}{d y^{\lfloor\alpha\rfloor+1}} f(y) ;
$$

for $\alpha \in \mathbb{N}_{+}$, the fractional derivative is the usual derivative (by analytical continuation in $\alpha$ ) and we have in the general case the identity:

$$
\mathrm{D}^{\alpha} \mathrm{D}^{-\alpha}=\mathrm{D}^{0}=\mathrm{Id}
$$

Then we have:

$$
\mathcal{G}_{v}^{(s)}(z)=\frac{1}{\Gamma(s)} \mathrm{D}^{s-1} \mathcal{G}_{v}(z) .
$$

It will useful later on to develop an inverse formula similar to the famous Plemelj inversion formula in the $s=1$ case:

$$
v(x)=\frac{1}{\pi} \Im \mathfrak{m} \lim _{\eta \searrow 0} \mathcal{G}_{v}(x-\mathrm{i} \eta)
$$

If we denote by:

$$
g^{(s)}(x):=\frac{1}{\pi} \mathfrak{I m} \lim _{\eta \searrow 0} \mathcal{G}_{v}^{(s)}(x-\mathrm{i} \eta)
$$

then taking the corresponding limit in (101) together with (102) and using the identity (100) yields:

$$
v(x)=\Gamma(s) \mathrm{D}^{1-s} g^{(s)}(x),
$$

which gives explicitly:

\footnotetext{
${ }^{8}$ Note that we are interested in functions that are regular at infinity but not necessarily near zero, hence we integrate to infinity and not from zero as it is more customary.
} 
- For $0<s<1$ :

$$
v(x):=-\int_{x}^{\infty} d y(y-x)^{s-1} \frac{d}{d y} g^{(s)}(y)
$$

- for $s>1$ :

$$
v(x)=(s-1) \int_{x}^{\infty} d y(y-x)^{s-2} g^{(s)}(y) .
$$

\subsection{Properties of the Markov Krein transform}

Taking (88) together with (91) and (95) we have that the MKT is linked to the original measure by:

$$
\mathcal{G}_{\mathcal{M}_{c, \mu}(z)}^{(c)}\left(z U_{\mu}^{[c]}(z) .\right.
$$

This relation and its application to different fields are explained in Kerov [44]. More explicitly we can write:

$$
\int_{J} d x \frac{\mathcal{M}_{c, \mu}(x)}{(z-x)^{c}}=\exp \left\{-c \int_{I} d x \log (z-x) \mu(x)\right\},
$$

which can also be seen as a non linear differential equation:

$$
\frac{d}{d z} \mathcal{G}_{\mathcal{M}_{c, \mu}}^{(c)}(z)+c \mathcal{G}_{\mathcal{M}_{c, \mu}}^{(c)}(z) \mathcal{G}_{\mu}(z)=0
$$

Since the functions on the LHS and RHS of (108) are equal and analytic on the complex plane except for the real line going from $-\infty$ to the right extremity of the support of their respective distribution and since for both functions crossing the branch cut on the left of the supports simply introduces a phase $e^{2 \mathrm{i} \pi c}$, we have necessarily equality between the support of the two distributions. Next using the formal series expansions (84) and (93) together with the definition of the normalized Jack polynomials in the high temperature regime (81), we can express the moments of the MKT $m_{k}\left(\mathcal{M}_{c, \mu}\right)=\int d x x^{k} \mathcal{M}_{c, \mu}(x)$ in terms of the moments $m_{k}(\mu)=\int d x x^{k} \mu(x)$ of the original measure:

$$
m_{k}\left(\mathcal{M}_{c, \mu}\right)=\frac{\Gamma(c) k !}{\Gamma(c+k)} \sum_{1 j_{1}+\cdots+k j_{k}=k} c^{j_{1}+\cdots+j_{k}} \prod_{i=1}^{k} \frac{m_{i}(\mu)^{j_{i}}}{i^{j_{i}} j_{i} !} .
$$

For completeness we give the inverse mapping, together with (85) and (93) at $s=1$, we have:

$$
m_{k}(\mu)=\frac{k}{c} \sum_{1 j_{1}+\cdots+k j_{k}=k}(-1)^{\sum_{i} j_{i}-1}\left(\sum_{i} j_{i}-1\right) ! \prod_{i}\left(\frac{\Gamma(c+i)}{\Gamma(c) i !}\right)^{j_{i}} \frac{m_{i}\left(\mathcal{M}_{c, \mu}\right)^{j_{i}}}{j_{i} !} .
$$

In particular, we have that the means $m_{1}$ of the two distributions are equal and the variances are linked by:

$$
m_{2}\left(\mathcal{M}_{c, \mu}\right)-m_{1}\left(\mathcal{M}_{c, \mu}\right)^{2}=\frac{1}{c+1}\left(m_{2}(\mu)-m_{1}(\mu)^{2}\right)
$$


As in the discrete case, the high temperature MKT has a smaller variance than the original distribution. It is still non zero in this formally $N \rightarrow \infty$ regime. In the limit $c \rightarrow \infty$ we recover the zero variance found for fixed $\beta$ and infinite $N$. From (108), one can show that a shift and a scaling applied to a density introduces the same shift and scaling to its MKT. Similarly, for an original symmetric distribution $\mu$, up to a shift we can fix the axis of symmetry to be the $x=0$ axis without loss of generality, then we have that the RHS of (108) is invariant under the symmetry $z \rightarrow-z$ and therefore so does the LHS, which implies that the MKT is also symmetric along the same axis. It turns out that if we assume furthermore the distribution $\mu$ to be unimodal (in addition to being symmetric), then its MKT is also unimodal with the same vertex [46] but unlike the previous properties, the converse is not true. Using the Taylor expansion in $c$ of the exponential function in (108), we have:

$$
1-c \int_{\mathbb{R}} d x \log (z-x) \mathcal{M}_{c, \mu}(x)+O\left(c^{2}\right)=1-c \int_{\mathbb{R}} d x \log (z-x) \mu(x)+O\left(c^{2}\right),
$$

from which we derive the limit:

$$
\lim _{c \rightarrow 0} \mathcal{M}_{c, \mu} \rightarrow \mu \text {. }
$$

Similarly taking the limit $c \rightarrow \infty$ in (112) we immediately find the other extreme case:

$$
\lim _{c \rightarrow \infty} \mathcal{M}_{c, \mu} \rightarrow \delta\left(x-m_{1}\right) .
$$

We now aim at finding an explicit expression for the distribution of the MKT. Taking the imaginary part of the RHS of (108) in the limit $\eta \searrow 0$ together with $z=x-\mathrm{i} \eta, x \in I$ and the behavior of the logarithm near the real axis, one can derive the following limit [45]:

$$
g^{(c)}(x)=\frac{1}{\pi} e^{-c \int d y \log |x-y| \mu_{A}(y)} \sin \left(\pi c \mu_{A}[x, \infty]\right) \quad(x \in I) .
$$

From which we get the density of the MKT with the proper inversion formula (105) for $c<1$ and (106) for $c>1$, while for $c=1$, we have directly $\mathcal{M}_{1, \mu}()=.g^{(1)}($.$) . In particular we have$ that the MKT density is absolutely continuous with respect to the Lebesgue measure. Next we give a few examples of the MKT of distributions that have already appeared before in the literature and that will be useful later on.

\subsection{Known Markov Krein transforms}

MKT of the Bernoulli distribution: Let us denote by

$$
\mu_{B(p)}(x):=(1-p) \delta(x-0)+p \delta(x-1),
$$

the Bernoulli distribution with probability of success $p$, then one can show $[46,47]$ that its MKT follows the law of a beta distribution $\beta(c p, c(1-p))$ so that we have:

$$
\mathcal{M}_{c, \mu_{B(p)}}(x)=\frac{\Gamma(c)}{\Gamma(c p) \Gamma(c(1-p))} x^{c p-1}(1-x)^{c(1-p)-1} \mathbb{I}_{[0,1]},
$$

where $\mathbb{I}$ is the indicator function. It is worth mentioning that the result can be derived by first looking at the finite $N$ case and then take the high temperature limit. The vector $\boldsymbol{a}=(0, \ldots, 0,1, \ldots, 1)$ of size $N$ with $p N$ non-zero values equal to 1 has a spectral distribution given by (117). By the symmetry (12), we can re-scale $\beta$ and $N$ by $p N$ accordingly so 
that the computation of the corresponding HCIZ integral boils down to the computation of a rank one normalized Jack polynomial which is given by [32]:

$$
\mathrm{g}_{k}^{\left(\frac{2}{\beta}\right)}(1,0, \ldots, 0)=\frac{\prod_{i=0}^{k-1}\left(\frac{\beta}{2}+i\right)}{k !} .
$$

Taking the high temperature regime in (9), we get that the $c$-HCIZ is given by:

$$
\mathcal{I}_{\mu_{B(p)}^{[c]}}(t)={ }_{1} F_{1}(c p, c, t)
$$

which is the moment generating function of (118).

MKT of the arcsine distribution: Another known example in closed form (see for example [46] and reference therein) is given when the original distribution is the arcsine distribution:

$$
\mu_{A s}(x):=\frac{1}{\pi \sqrt{x(1-x)}} \mathbb{I}_{[0,1]},
$$

then one may show that its MKT follows the law of a beta distribution $\beta\left(c+\frac{1}{2}, c+\frac{1}{2}\right)$ :

$$
\mathcal{M}_{c, \mu_{A s}}(x)=\frac{\Gamma(2 c+1)}{\Gamma\left(c+\frac{1}{2}\right)^{2}}(x(1-x))^{c-\frac{1}{2}} \mathbb{I}_{[0,1]},
$$

this can be checked by computing the LHS and RHS of (108) with the corresponding measures.

MKT of the uniform distribution: If we now take the original distribution to be the uniform distribution on $[0,1]$ :

$$
\mu_{U}:=\mathbb{I}_{[0,1]}
$$

then by (116) we have:

$$
g^{(c)}(x)=\frac{e^{c}}{\pi}(1-x)^{-c(1-x)} x^{-c x} \sin (\pi c(1-x)) \mathbb{I}_{[0,1]},
$$

which gives in particular the density for $c=1$ of the corresponding MKT transform. For $c<1$ and $c>1$, one needs to use the formula (105) and (106) but no analytical expression is known.

MKT of the Cauchy distribution: For every $c>0$, the Markov-Krein transform of a Cauchy distribution with parameters $x_{0}$ and $b$ :

$$
\mu_{C_{x, b}}(x):=\frac{b}{\pi\left(b^{2}+\left(x-x_{0}\right)^{2}\right)},
$$

is again a Cauchy distribution with the same parameters (which can be seen by computing LHS and RHS of (108), see for example [45, 48]). 


\subsection{Inverting the Markov-Krein transform}

For a given measure $\mu$ and a positive real $c$, we have seen that there is always a unique welldefined probability measure which is its MKT. It is natural to ask the reverse question: for a probability measure $v$ and a positive real $c$, can we find and express a measure $\mu$ such that $v$ is the MKT of $\mu$ ? The measure $\mu$ will therefore be the inverse Markov-Krein Transform (IMKT) of $v$ and denoted by $\mathcal{M}_{c, v}^{-1}$. Kerov proved that the IMKT of a probability measure always exists and is unique but not necessarily positive. The image of the set of probability measure by the IMKT is discussed in details in [44]. We aim now at expressing the measure of the IMKT given the density $v($.$) . From (86) and (108), one has:$

$$
\begin{aligned}
& \mathcal{M}_{c, v}^{-1}(x)=-\frac{1}{c \pi} \frac{d}{d x} \lim _{\eta \searrow 0} \mathfrak{I m} \log \left\{\mathcal{G}_{v}^{(c)}(x-\mathrm{i} \eta)\right\}, \\
& \mathcal{M}_{c, v}^{-1}(x)=-\frac{1}{c \pi} \frac{d}{d x} \arctan \left(\frac{\lim _{\eta \searrow 0} \mathfrak{I m} \mathcal{G}_{v}^{(c)}(x-\mathrm{i} \eta)}{\lim _{\eta \searrow 0} \mathfrak{R e} \mathcal{G}_{v}^{(c)}(x-\mathrm{i} \eta)}\right),
\end{aligned}
$$

where we have used that the imaginary part of the logarithm is (up to an irrelevant constant) the arctan function of the ratio of the imaginary and real part of its argument. If we know the generalized Stieltjes transform of $v$, (127) can be used directly. We can also use the link between the generalized and the standard Stieltjes transform via the fractional derivative to express the IMKT measure more directly as a function of $v$. From Section 4.2, we already know that:

$$
\lim _{\eta \searrow 0} \mathfrak{I m} \mathcal{G}_{v}^{(c)}(x-\mathrm{i} \eta)=\frac{\pi}{\Gamma(c)} \mathrm{D}^{c-1} v(x) .
$$

Similarly for the real part, since for $c=1$ we have:

$$
\frac{1}{\pi} \lim _{\eta \searrow 0} \mathfrak{R e} \mathcal{G}_{v}(x-\mathrm{i} \eta)=\mathcal{H}_{v}(x)
$$

where

$$
\mathcal{H}_{\nu}(x):=\frac{1}{\pi} \mathrm{P} . \mathrm{V} \int_{I} d y \frac{v(y)}{x-y},
$$

is the Hilbert transform of the measure $v$ and P.V indicates that the integral has to be understood as a Cauchy principal value integral, we find:

$$
\lim _{\eta \searrow 0} \mathfrak{R e} \mathcal{G}_{v}^{(c)}(x-\mathrm{i} \eta)=\frac{\pi}{\Gamma(c)} \mathrm{D}^{c-1} \mathcal{H}_{v}(x) .
$$

Equation (127) can therefore be written as:

$$
\mathcal{M}_{c, v}^{-1}(x)=-\frac{1}{c \pi} \frac{d}{d x} \arctan \left(\frac{\mathrm{D}^{c-1} v(x)}{\mathcal{H}_{\mathrm{D}^{c-1} v}(x)}\right)
$$

where we have used the fact the the fractional derivative and the Hilbert transform are both linear kernel operators and therefore commute. If the density $\mathcal{M}_{c, v}^{-1}$ is continuous, this reads:

$$
\mathcal{M}_{c, v}^{-1}(x)=\frac{1}{c \pi} \frac{\mathcal{H}_{\mathrm{D}^{c} v}(x) \mathrm{D}^{c-1} v(x)-\mathcal{H}_{\mathrm{D}^{c-1} v}(x) \mathrm{D}^{c} v(x)}{\left(\mathcal{H}_{\mathrm{D}^{c-1} v}(x)\right)^{2}+\left(\mathrm{D}^{c-1} v(x)\right)^{2}} .
$$


One has to be careful when applying (132) or (133): while the density of the IMKT is defined on the same support as that of the measure $v$, the fractional derivative is a non-local operator and should be compute for all $x<a_{\max }$ before computing its Hilbert transform. Note as well that our definition of fractional derivative uses a boundary condition at infinity rather than the more usual boundary at zero. For these reasons these formulas are difficult to use in practice, except for integer $c$ where the fractional derivative reduces to the usual derivative. We finish this section with several examples of IMKT:

IMKT of the standard Gaussian distribution: For

$$
v_{G}(x):=\frac{e^{-\frac{x^{2}}{2}}}{\sqrt{2 \pi}},
$$

a standard Gaussian distribution, then one has that the IMKT is given by the so-called AskeyWimp-Kerov distribution:

$$
\mathcal{M}_{c, v_{G}}^{-1}(x)=\frac{1}{\sqrt{2 \pi} \Gamma(c+1)} \frac{1}{\left|D_{-c}(\mathrm{i} x)\right|^{2}},
$$

where $D_{-c}($.$) is a parabolic cylinder function defined by:$

$$
D_{-c}(z):=\frac{e^{-\frac{z^{2}}{4}}}{\Gamma(c)} \int_{0}^{\infty} d x e^{-z x-\frac{x^{2}}{2}} x^{c-1} .
$$

It has zero mean and variance $c+1$. This distribution was first obtained by Kerov [44], while the distribution had first appeared in [49] as the distribution whose orthogonal polynomials are the associated Hermite polynomials, since then it has appeared also in RMT ensemble at high temperature $[12,14]$. This distribution is a continuous interpolation between the Gaussian distribution at $c=0$ and the unnormalized (infinite variance) semi-circle distribution at $c \rightarrow \infty$.

IMKT of the gamma distribution: The gamma distribution with parameter $(k, \theta)$ is defined by the probability density:

$$
v_{\gamma(k, \theta)}(x):=\frac{e^{-\frac{x}{\theta}} x^{k-1}}{\Gamma(k) \theta^{k}}
$$

Since the parameter $\theta$ is a scale parameter, we can fix it to $\theta=1$ without loss of generality thanks to the scaling property of Section 4.3 and we simply denote by $v_{\gamma(k)}$ the gamma distribution in this case. Since the support of the measure is $(0, \infty)$, it will be more convenient to characterize it by its Laplace transform:

$$
\mathbb{E}_{X \sim \gamma(k)}\left[e^{-t X}\right]=(1+t)^{-k}
$$

We can use the following identity for the Tricomi function $\Psi($.$) :$

$$
\Psi(c, c+1-k ; z):=\frac{1}{\Gamma(c)} \int_{0}^{\infty} d t e^{-z t} t^{c-1}(1+t)^{-k} \quad(\Re \mathfrak{k} z>0),
$$

taking care of the branch cut on the negative real axis of the Tricomi function and using property (89), we have for $z \in \mathbb{C} \backslash \mathbb{R}_{+}$, that up to an irrelevant multiplicative constant:

$$
U_{\mathcal{M}_{c, \nu}^{-1}}^{[c]}(z) \propto \Psi(c, c+1-k ;-z) .
$$


Since we have:

$$
\frac{d}{d z} \Psi(c, c+1-k ;-z)=c \Psi(c+1, c+2-k ;-z) .
$$

Using (85) we find that the corresponding Stieltjes transform is given by:

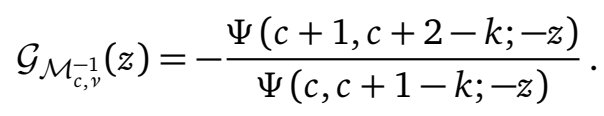

It turns out that a similar Stieltjes transform had already appear in the RMT literature in a different context $[13,17]$ from which we can immediately get the limiting density:

$$
\mathcal{M}_{c, v}^{-1}(x)=\frac{1}{\Gamma(c+1) \Gamma(k)} \frac{x^{k-c-1} e^{-x}}{\left|\Psi\left(c, c+1-k ; e^{\mathrm{i} \pi^{-}} x\right)\right|^{2}} \mathbb{I}_{x>0}+\mathbb{I}_{c>k} \frac{c-k}{c} \delta(x-0) .
$$

Crossing the branch cut of the Tricomi function introduces a change in the sign of the imaginary part of the function so that the function $\left|\Psi\left(\alpha_{1}, \alpha_{2} ;.\right)\right|$ can be continued analytically to all $\mathbb{C}$. The density in (143) does not depend on the choice of the branch cut, in particular one could have taken instead $e^{\mathrm{i} \pi^{+}}$in the argument of the Tricomi function. The case $\theta \neq 1$ is then obtained by dilatation and we have:

$$
\mathcal{M}_{c, v}^{-1}(x)=\frac{\theta^{c-k}}{\Gamma(c+1) \Gamma(k)} \frac{x^{k-c-1} e^{-\frac{x}{\theta}}}{\left|\Psi\left(c, c+1-k ;-\frac{x}{\theta}\right)\right|^{2}} \mathbb{I}_{x>0}+\mathbb{I}_{c>k} \frac{c-k}{c} \delta(x-0) .
$$

This distribution has mean $k \theta$ and variance $k \theta^{2}(c+1)$, it interpolates between the gamma distribution (at $c=0$ ) and the (rescaled) Marčenko-Pastur distribution. To recover the standard Marčenko-Pastur with aspect ratio $q$, one has to take the limit $c \rightarrow \infty$ with $k \rightarrow q c$ and $\theta \rightarrow(q c)^{-1}$.

IMKT of the beta distribution: It is natural to ask if one can find a positive measure for the IMKT of a beta distribution since it is the third classical ensemble after the Gaussian and the gamma distribution. In Section 4.4, we saw that certain beta distributions are the MKT of the Bernouilli and arcsine distributions, but for a general beta distribution its IMKT is not always a positive measure. We will show this by finding a triplet $(c, a, b)$ where $(a, b)$ are the parameters of the beta distribution, such that the IMKT is not positive.

The moment generating function of the beta distribution $\beta(a, b)$ is given by:

$$
\mathbb{E}_{X \sim \beta(a, b)}\left[e^{t X}\right]={ }_{1} F_{1}(a, a+b ; t) .
$$

We have from (88) and (91) that the corresponding IMKT satisfies:

$$
U_{\mathcal{M}_{c, \nu}^{-1}}^{[c]}(z)=\frac{1}{\Gamma(c)} \int_{0}^{\infty} d t e^{-z t} t^{c-1}{ }_{1} F_{1}(a, a+b ; t),
$$

by the classical identity between the hypergeometric functions:

$$
{ }_{2} F_{1}(a, c, a+b ; z)=\frac{1}{\Gamma(c)} \int_{0}^{\infty} d t e^{-t} t^{c-1}{ }_{1} F_{1}(a, a+b ; z t),
$$

we find:

$$
U_{\mathcal{M}_{c, v}^{-1}}^{[c]}(z)=z^{-c}{ }_{2} F_{1}(a, c, a+b ; 1 / z) .
$$


From (85) together with the identity for the derivative of the hypergeometric function ${ }_{2} F_{1}($.$) :$

$$
\frac{d}{d x}{ }_{2} F_{1}(\alpha, \beta, \gamma ; x)=\frac{\alpha \beta}{\gamma}{ }_{2} F_{1}(\alpha+1, \beta+1, \gamma+1 ; x),
$$

we get, after simplifications,

$$
\mathcal{G}_{\mathcal{M}_{c, v}^{-1}}(z)=\frac{1}{z}+\frac{a_{2} F_{1}(a+1, c+1, a+b+1 ; 1 / z)}{(a+b) z^{2}{ }_{2} F_{1}(a, c, a+b ; 1 / z)} .
$$

This expression is the Stieltjes transform of the IMKT of a beta distribution with arbitrary parameters $a$ and $b$, in particular we recover the Stieltjes transform of the Bernoulli (117) for $a=c p$ and $b=c(1-p)$ and the arcsine law (121) for $a=b=c+1 / 2$.

As an explicit example of a non positive IMKT we fix $c=2, a=b=\frac{1}{2}$, in this case the expression simplifies considerably and we have:

$$
\begin{aligned}
\mathcal{G}_{\mathcal{M}_{c, v}^{-1}}(z) & =\frac{3-8 z+8 z^{2}}{4 z-12 z^{2}+8 z^{3}}, \\
\mathcal{G}_{\mathcal{M}_{c, v}^{-1}}(z) & =\frac{3}{4} \frac{1}{z-1}+\frac{3}{4} \frac{1}{z}-\frac{1}{2 z-1} .
\end{aligned}
$$

From which we find that the IMKT is the discrete distribution:

$$
\mathcal{M}_{c, v}^{-1}(x)=\frac{3}{4} \delta(x-0)-\frac{1}{2} \delta\left(x-\frac{1}{2}\right)+\frac{3}{4} \delta(x-1),
$$

and hence it is not a positive measure.

\section{5 c-convolution}

\section{$5.1 \quad c$-convolution as convolution of Markov-Krein transforms}

The HCIZ integral is multiplicative for the free convolution for $\beta>0$, in the limit $N \rightarrow \infty$, and multiplicative for the classical convolution at $\beta=0$. It is natural to construct a new convolution, which we call the c-convolution and denote it by $\oplus_{c}$, for which the HCIZ in the high temperature regime $\frac{N \beta}{2} \rightarrow c$ of the previous section is multiplicative. Using (91) this is equivalent to saying that our $c$-convolution corresponds to a classical convolution in the Markov-Krein space. This statement can be summarized by the following scheme:

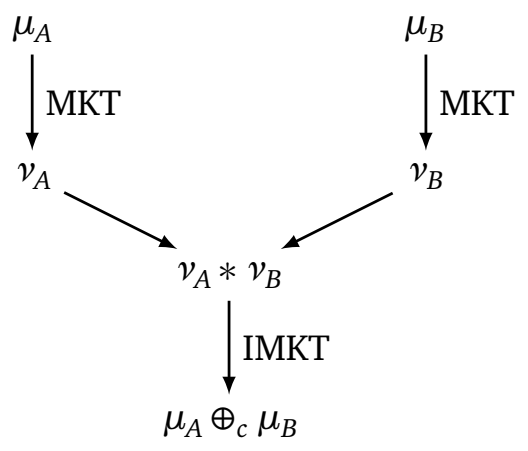

The $c$-convolution

- is commutative: $\mu_{A} \oplus_{c} \mu_{B}=\mu_{B} \oplus_{c} \mu_{A}$, 
- is associative: $\mu_{A} \oplus_{c}\left(\mu_{B} \oplus_{c} \mu_{C}\right)=\left(\mu_{A} \oplus_{c} \mu_{B}\right) \oplus_{c} \mu_{C}$,

- is well behaved with respect to shift: $\mu_{A} \oplus_{c} \delta_{x_{0}}=\mu_{A}\left(.-x_{0}\right)$,

- preserves symmetric measures: if two distributions are symmetric with respect to their means then their $c$-convolution is also symmetric with respect to its mean,

- is additive for the means and the variances:

$$
m_{2}\left(\mu_{A} \oplus_{c} \mu_{B}\right)-\left(m_{1}\left(\mu_{A} \oplus_{c} \mu_{B}\right)\right)^{2}=m_{2}\left(\mu_{A}\right)-m_{1}\left(\mu_{A}\right)^{2}+m_{2}\left(\mu_{B}\right)-m_{1}\left(\mu_{B}\right)^{2},
$$

- admits the $\operatorname{limits}_{\lim _{c \rightarrow 0}} \mu_{A} \oplus_{c} \mu_{B}=\mu_{A} * \mu_{B}$ and $\lim _{c \rightarrow \infty} \mu_{A} \oplus_{c} \mu_{B}=\mu_{A} \boxplus \mu_{B}$.

All these properties are derived immediately from the properties of the Markov-Krein transform of Section 4.3. In the general setting, the c-convolution is defined on the set of the images of the IMKT described by Kerov [44] (see also [45]), it is an open and important question to know whether the $c$-convolution is stable for probability measures, that is, the $c$-convolution of two probability measures is again a probability measure. We emphasize that this convolution is well suited for numerical simulations since the operations to compute the MKT on the one hand, namely (116) together with (105) or (106) and the ones to compute the IMKT with (133) or with (126) and the definition (92) can all be approximated numerically. We have illustrated the results of the $c$-convolution of several well-known examples of distributions in the classical and free worlds in Fig. 1.

\section{2 c-cumulants}

The c-convolution being defined, the next step is to define the corresponding c-cumulants which we denote by $\kappa_{k}^{(c)}$. Following Lehner [50], the $c$-cumulants must satisfy

- additivity: $\kappa_{k}^{(c)}\left(\mu_{A} \oplus_{c} \mu_{B}\right)=\kappa_{k}^{(c)}\left(\mu_{A}\right)+\kappa_{k}^{(c)}\left(\mu_{B}\right)$,

- homogeneity: $\kappa_{k}^{(c)}\left(\frac{1}{\lambda} \mu_{A}(\dot{\bar{\lambda}})\right)=\lambda^{k} \kappa_{k}^{(c)}\left(\mu_{A}\right)$,

- $\kappa_{k}^{(c)}$ is a polynomial in the first $k$ moments with leading term $m_{k}$.

By construction of the $c$-convolution we have that the (classical) cumulants of the MKT are additive (and of course homogeneous) for the $c$-convolution. Their leading term is given by the $k^{\text {th }}$ moment of the MKT and not the $k^{\text {th }}$ moment of the original distribution, so that we need to compute the term $C_{k, c}$ in the development:

$$
m_{k}\left(\mathcal{M}_{c, \mu}\right)=C_{k, c} m_{k}(\mu)+\ldots
$$

Using (110) this is given by:

$$
C_{k, c}=\frac{\Gamma(c+1)(k-1) !}{\Gamma(c+k)},
$$

hence dividing by $C_{k, c}$ the classical cumulant of the MKT we get the $c$-cumulant, from which we derive that they satisfy the following equation:

$$
\log \left(1+\sum_{k=1}^{\infty} \frac{m_{k}\left(\mathcal{M}_{c, \mu}\right)}{k !} t^{k}\right)=\sum_{k=1}^{\infty} \frac{\Gamma(c+1)}{\Gamma(c+k) k} \kappa_{k}^{(c)} t^{k} .
$$




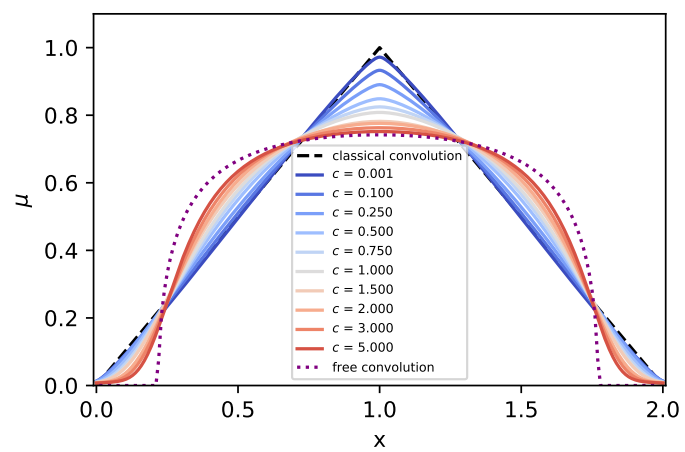

(a)

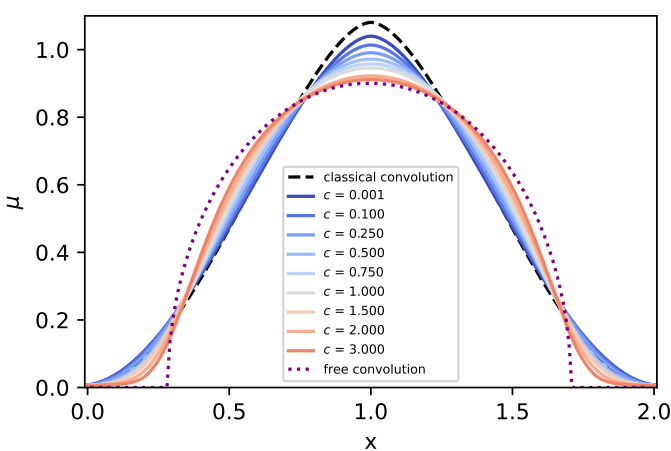

(b)

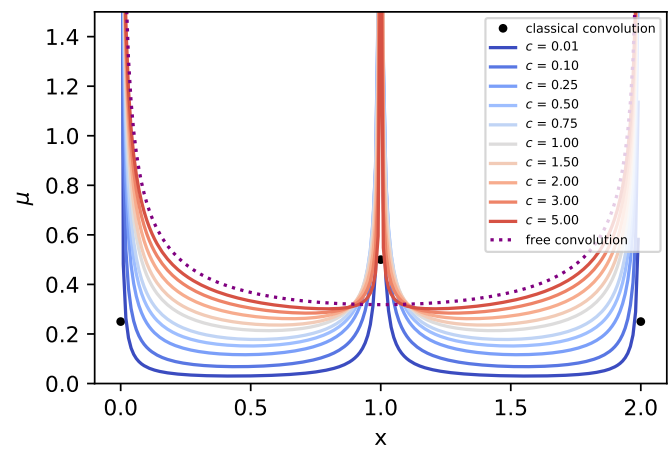

(c)

Figure 1: Plots of numerical approximations of the c-convolution of the uniform distribution (a) the semi-circle (b) and the symmetric Bernoulli distribution (c) with itself for different values of $c$. The dashed line corresponds to the classical convolution and the dotted line to the free convolution limiting cases.

For completeness we give the cumulant-moment expression:

$$
\kappa_{k}^{(c)}=\frac{\Gamma(c+k) k}{c} \sum_{1 j_{1}+\cdots+k j_{k}}(-\Gamma(c))^{\sum_{i} j_{i}-1} \frac{\left(\sum_{i} j_{i}-1\right) !}{\prod_{i} j_{i} ! \Gamma(c+i)^{j_{i}}} \prod_{i}\left(\sum_{1 l_{1}+\cdots+i l_{i}=i} c^{\sum l_{n}} \prod_{n} \frac{m_{n}^{l_{n}}}{n^{l_{n}} l_{n} !}\right)^{j_{i}},
$$

from which we can derive the first cumulant-moment relations:

$$
\begin{aligned}
\kappa_{1}^{(c)} & =m_{1}, \\
\kappa_{2}^{(c)} & =m_{2}-m_{1}^{2}, \\
\kappa_{3}^{(c)} & =m_{3}-3 m_{2} m_{1}+2 m_{1}^{3}, \\
\kappa_{4}^{(c)} & =m_{4}-4 m_{3} m_{1}-\left(2+\frac{1}{c+1}\right) m_{2}^{2}+\left(10+\frac{2}{c+1}\right) m_{2} m_{1}^{2}-\left(5+\frac{1}{c+1}\right) m_{1}^{4} \\
\kappa_{5}^{(c)} & =m_{5}-5 m_{4} m_{1}-5\left(1+\frac{1}{c+1}\right) m_{3} m_{2}+\left(15+\frac{5}{c+1}\right) m_{3} m_{1}^{2}+15\left(1+\frac{1}{c+1}\right) m_{2}^{2} m_{1} \\
& -\left(35+\frac{25}{c+1}\right) m_{2} m_{1}^{3}+\left(14+\frac{10}{c+1}\right) m_{1}^{5} .
\end{aligned}
$$

In particular when the first moment $m_{1}=0$, we have that the $4^{\text {th }}$ cumulant is given by:

$$
\kappa_{4}^{(c)}=m_{4}-m_{2}^{2}\left(\frac{2 c+3}{c+1}\right),
$$


from which we see that the value $c=1$ corresponds to the midpoint between the classical and free case. Similarly we can obtain the moments in terms of terms of the $c$-cumulants, we only give here the first five moment-c-cumulant relations:

$$
\begin{aligned}
m_{1} & =\kappa_{1}^{(c)} \\
m_{2} & =\kappa_{2}^{(c)}+\left(\kappa_{1}^{(c)}\right)^{2}, \\
m_{3} & =\kappa_{3}^{(c)}+3 \kappa_{2}^{(c)} \kappa_{1}^{(c)}+\left(\kappa_{2}^{(c)}\right)^{3}, \\
m_{4} & =\kappa_{4}^{(c)}+4 \kappa_{3}^{(c)} \kappa_{1}^{(c)}+\left(2+\frac{1}{c+1}\right)\left(\kappa_{2}^{(c)}\right)^{2}+6 \kappa_{2}^{(c)}\left(\kappa_{1}^{(c)}\right)^{2}+\left(\kappa_{1}^{(c)}\right)^{4}, \\
m_{5} & =\kappa_{5}^{(c)}+5 \kappa_{4}^{(c)} \kappa_{1}^{(c)}+\left(5+\frac{5}{c+1}\right) \kappa_{3}^{(c)} \kappa_{2}^{(c)}+10 \kappa_{3}^{(c)}\left(\kappa_{1}^{(c)}\right)^{2}+\left(10+\frac{5}{c+1}\right)\left(\kappa_{2}^{(c)}\right)^{2} \kappa_{1}^{(c)} \\
& +10 \kappa_{2}^{(c)}\left(\kappa_{1}^{(c)}\right)^{3}+\left(\kappa_{1}^{(c)}\right)^{5} .
\end{aligned}
$$

Remark: If one writes back $c=\frac{N \beta}{2}$ and set $\beta=-2$, one recovers the combinatorial formula for the finite free cumulants of the finite free convolution of Section 3, see [42].

\section{$5.3 \quad c$-central limit theorem and related distributions}

Let $\mu$ be a measure with zero mean and unit variance, then we look at the following $c$-Central Limit Theorem (c-CLT):

$$
\mu_{G}^{[c]}(.):=\lim _{T \rightarrow \infty} \sqrt{T} \mu(\sqrt{T} .)^{\oplus_{c} T},
$$

where.$^{\oplus_{c} T}$ indicates that we do the $c$-convolution of the measure $\mu T$ times. The $\mu_{G}^{[c]}($.$) is the$ $c$-Gaussian distribution, which is the unit variance Askey Wimp Kerov distribution of equation (135), given by:

$$
\mu_{G}^{[c]}(x)=\frac{\sqrt{c+1}}{\sqrt{2 \pi} \Gamma(c+1)} \frac{1}{\left|D_{-c}(\mathrm{i} \sqrt{c+1} x)\right|^{2}} .
$$

Indeed, the Markov-Krein transform of $\mu_{i}$ is a distribution with mean zero and variance $\frac{1}{c+1}$, since $c$-convolution corresponds to classical convolution in the Markov-Krein space, we have by the classical central limit theorem that the limiting distribution is the IMKT transform of the Gaussian distribution with variance $\frac{1}{c+1}$. But we know from the previous example that the IMKT of the standard Gaussian distribution is given by (135), so by the scaling property derived in Section 4.3 we have the desired result. By construction, the orthogonal polynomials of the $c$-Gaussian distribution continuously interpolate between the Hermite polynomials of the (classical) Gaussian and the Chebyshev polynomials of the second kind of the semi-circle distribution and are known as the (rescaled) associated Hermite polynomials, see [49]. As illustrated in Fig 2, this distribution is a continuous interpolation between the standard Gaussian distribution and the semi-circle distribution, in accordance with the properties of the $c$-convolution.

c-cumulants: Since the MKT of the $c$-Gaussian is a Gaussian, we find immediately from results of the previous section, that the cumulants of the $c$-Gaussian are defined by:

$$
\kappa_{k}^{(c)}=1 \delta_{k, 2},
$$

where $\delta_{k, 2}=1$ if $k=2$ and zero otherwise, which is expected from the limiting distribution of a CLT. 


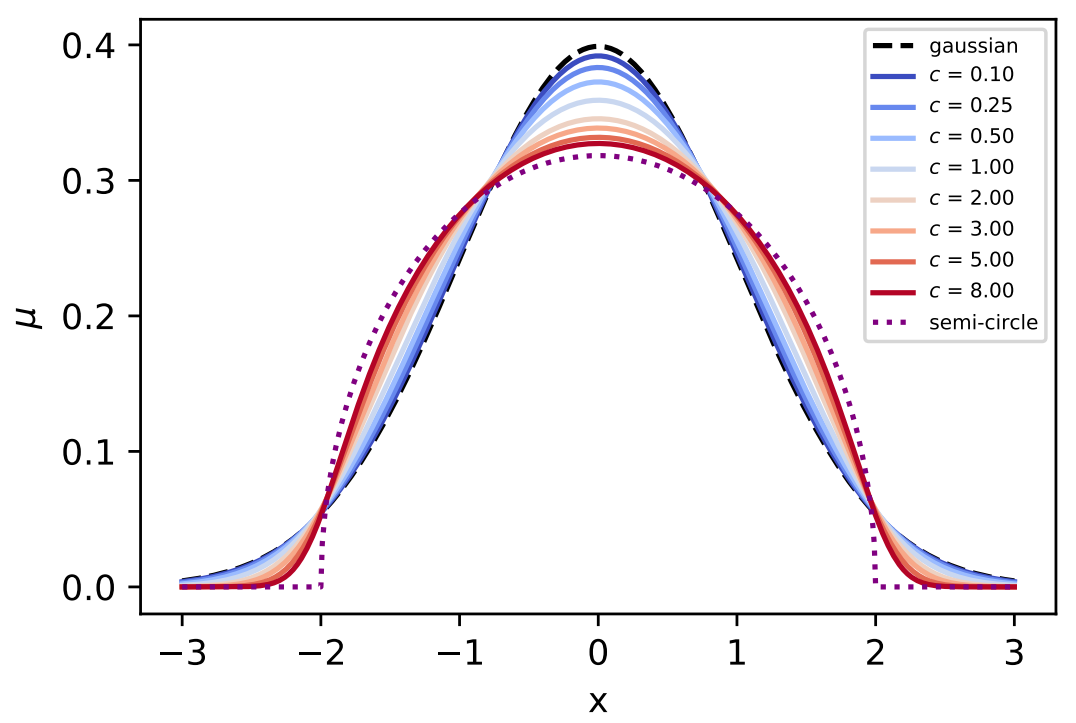

Figure 2: Plots of the $c$-Gaussian distribution defined in (161) for different values of $c$, the dashed lined corresponds to the classical limiting case and the doted line the free limiting case.

Infinite divisibility and the gamma Marčenko-Pastur crossover: In this section, we would like to interpolate between the gamma and Marčenko-Pastur (MP) distribution using their properties under convolution.

We consider the ensemble of gamma distributions (137) parameterized by their mean $k \theta$ and variance $k \theta^{2}$ and the (scaled) MP distributions of mean $\theta$ and variance $q \theta^{2}$ defined by:

$$
\mu_{M P(q, \theta)}(x):=\left(1-\frac{1}{q}\right) \delta(x-0) \mathbb{I}_{q>1}+\frac{\sqrt{\left(x_{+}-x\right)\left(x-x_{-}\right)}}{2 \pi q \theta x},
$$

where $x_{ \pm}=\theta(1 \pm \sqrt{q})^{2}$. The distributions in both ensemble are infinitely divisible (under classical or free convolution respectively) and are closed under scaling and convolution. Multiple families of law satisfy these two conditions; in order to uniquely determine the gamma and MP distribution we need to specify at least one member of the family: the square-Gaussian (or square semi-circle for MP). Indeed any gamma (MP) distribution can be obtained by scaling, convolution and convolution roots of the square-Gaussian (square-semi-circle), i.e. the random variable $y=x^{2}$ where $x$ is a unit centered Gaussian (semi-circle) random variable, it corresponds to a gamma distribution with $\theta=2, k=\frac{1}{2}$ (MP with $\theta=1, q=1$ ).

For any $c$, the $c$-gamma distributions given by (144) are infinitely divisible and closed under the $c$-convolution. Indeed, the c-convolution is defined as the convolution of MKTs and the MKT of a c-gamma is a gamma distribution (by construction) which is infinitely divisible and closed under convolution. For a given mean and variance the $c$-gamma tends to the gamma and Marčenko-Pastur distribution in the limit $c \rightarrow 0$ and $c \rightarrow \infty$ respectively. Let's see whether the $c$-gamma family also contains the squared c-Gaussian whose distribution is given by

$$
\rho^{(c)}(x):=\frac{1}{\sqrt{x}} \mu_{G}^{[c]}(\sqrt{x})=\frac{\sqrt{c+1}}{\sqrt{2 \pi} \Gamma(c+1)} \frac{x^{-\frac{1}{2}}}{\left|D_{-c}(\mathrm{i} \sqrt{(c+1) x})\right|^{2}},
$$

by property of the parabolic cylinder function, we have:

$$
D_{-s}(z)=2^{-s / 2} e^{\frac{z^{2}}{4}} \Psi\left(\frac{s}{2}, \frac{1}{2} ; \frac{z^{2}}{2}\right) .
$$




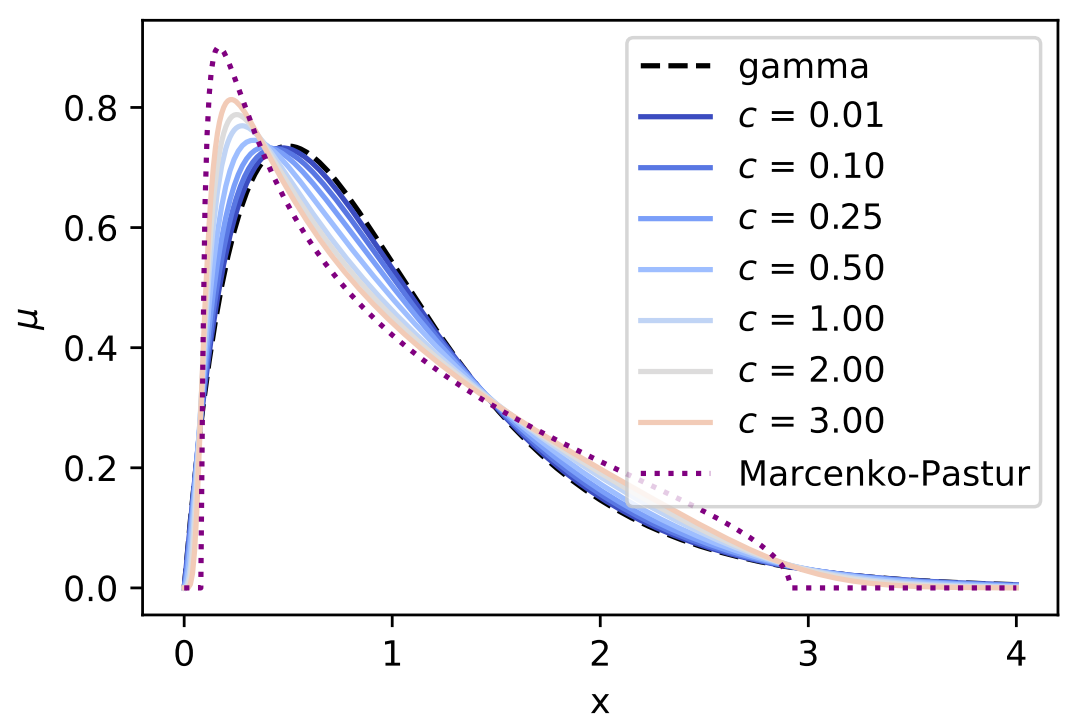

Figure 3: Plots of the c-gamma with mean 1 and variance $\frac{1}{2}$ for different values of $c$, the dashed lined corresponds to the classical limiting case and the doted line the free limiting case

Since we are taking the absolute value, we can again extend this formula near the branch cut, from which we have:

$$
\rho^{(c)}(x)=\frac{2^{c} \sqrt{c+1}}{\sqrt{2 \pi} \Gamma(c+1)} \frac{x^{-\frac{1}{2}} e^{-\frac{c+1}{2} x}}{\left|\Psi\left(\frac{c}{2}, \frac{1}{2} ; e^{i} \pi^{-\frac{c+1}{2}} x\right)\right|^{2}} \mathbb{I}_{x>0},
$$

where again we could have taken $e^{\mathrm{i} \pi \pi^{+}}$in the argument of the Tricomi function without changing the result. We recognize a $\tilde{c}$-gamma distribution (144) with parameters $\tilde{c}=c / 2, \theta=2 /(c+1)$ and $k=(c+1) / 2$. The normalizing constants look superficially different but they are indeed equal as they should be. Note that $k>\tilde{c}$ so this law doesn't have a mass at zero. The first two moments of both laws obviously match and are given by $\mu=1$ and $\sigma^{2}=(\tilde{c}+1) /(\tilde{c}+1 / 2)=(c+2) /(c+1)$.

So the $c$-gamma family contains a squared $c$-Gaussian but for a $c$ twice as large. This is still consistent with the $c$-gamma distribution interpolating between the standard gamma and MP distributions as when $c$ goes to either zero or infinity the $2 c$-Gaussian and the $c$-Gaussian become identical.

We have plotted in Fig 3, the distribution $\mu_{\gamma}^{[c]}($.) for different values of $c$.

It would be interesting to know whether one can construct explicitly a positive measure by replacing the $2 c$-Gaussian by the $c$-Gaussian. If such a construction exists it would yield a different interpolation between the gamma and the MP than the c-gamma considered here.

$c$-stability of the Cauchy distribution: By the $c$-CLT, we have that the $c$-Gaussian is $c$-stable. Another example of a $c$-stable distribution is given by the Cauchy distribution, since we know that it is a fixed point for both the MKT and the classical convolution, this writes simply:

$$
\mu_{C_{x_{1}, b_{1}}} \oplus_{c} \mu_{C_{x_{2}, b_{2}}}=\mu_{C_{x_{3}, b_{3}}},
$$

where the Cauchy distribution is defined in (125) and $x_{3}=x_{1}+x_{2}$ and $b_{3}=b_{1}+b_{2}$. 


\section{$5.4 \quad c$-Poisson limit theorem}

Another classical limit theorem is the Poisson central limit theorem which concerns limit of sum of independent Bernoulli random variables with a probability of success that goes to zero at a speed $\frac{1}{N}$ :

$$
\lim _{T \rightarrow \infty}\left(\left(1-\frac{\lambda}{T}\right) \delta(x-0)+\frac{\lambda}{T} \delta(x-a)\right)^{* T}=\frac{1}{a} \mu_{\mathrm{Poi}(\lambda)}\left(\frac{x}{a}\right) \quad(\text { for } a>0)
$$

where $\mu_{\mathrm{Poi}(\lambda)}(x)=\sum_{k=0}^{\infty} e^{-\lambda} \frac{\lambda^{k}}{k !} \delta(x-k)$ is the Poisson distribution. This limit theorem admits a free counterpart:

$$
\lim _{T \rightarrow \infty}\left(\left(1-\frac{\lambda}{T}\right) \delta(x-0)+\frac{\lambda}{T} \delta(x-a)\right)^{\boxplus T}=\frac{1}{a \lambda} \mu_{M P\left(\frac{1}{\lambda}\right)}\left(\frac{x}{a \lambda}\right) \quad(\text { for } a>0)
$$

where $\mu_{M P(q)}$ is the unit mean Marčenko-Pastur distribution, defined in (163) with $\theta=1$. In this subsection we aim at developing the $c$-counterpart of these theorems, whose limiting objects will interpolate between the Poisson and the re-scaled Marčenko-Pastur distribution. We know from (118), that the Markov-Krein transform of the Bernoulli distribution of probability of success $p$ is the beta distribution $\beta(c p, c(1-p))$. Since again $c$-convolution corresponds to classical convolution in the MK space, we first need to determine the limiting distribution of:

$$
v(.):=\lim _{T \rightarrow \infty}\left(\frac{1}{a} \beta\left(\frac{c \lambda}{T}, \frac{c(T-\lambda)}{T}\right)(\dot{\bar{a}})\right)^{* T},
$$

and then take the IMKT. This kind of distribution does not seem to have appeared before in the literature and we will characterize it with its moment generating function (as no closed form is known). The moment generating function of the beta distribution is given by (145), so that we have:

$$
\begin{aligned}
& \mathbb{E}_{X \sim \nu}\left[e^{t X}\right]=\lim _{T \rightarrow \infty}{ }_{1} F_{1}\left(\frac{c \lambda}{T}, c ; a t\right)^{T}, \\
& \mathbb{E}_{X \sim v}\left[e^{t X}\right]=\lim _{T \rightarrow \infty}\left(1+\frac{\sum_{k=1}^{\infty} \frac{\lambda \Gamma(c+1)}{\Gamma(c+k) k}(a t)^{k}}{T}+O\left(\frac{1}{T^{2}}\right)\right)^{T} .
\end{aligned}
$$

Next we use:

$$
{ }_{2} F_{2}(\{1,1\},\{2, c+1\} ; t)=\sum_{k=0}^{\infty} \frac{\Gamma(c+1)}{\Gamma(c+k+1)(k+1)} t^{k}=\frac{1}{t} \sum_{k=1}^{\infty} \frac{\Gamma(c+1)}{\Gamma(c+k) k} t^{k},
$$

where ${ }_{2} F_{2}$ is the hypergeometric function. Together with the classical limit identity for the exponential:

$$
e^{x}=\lim _{T \rightarrow \infty}\left(1+\frac{x}{T}\right)^{T}
$$

we get:

$$
\mathbb{E}_{X \sim v}\left[e^{t X}\right]=\exp \left\{a \lambda t_{2} F_{2}(\{1,1\},\{2, c+1\} ; a t)\right\}
$$




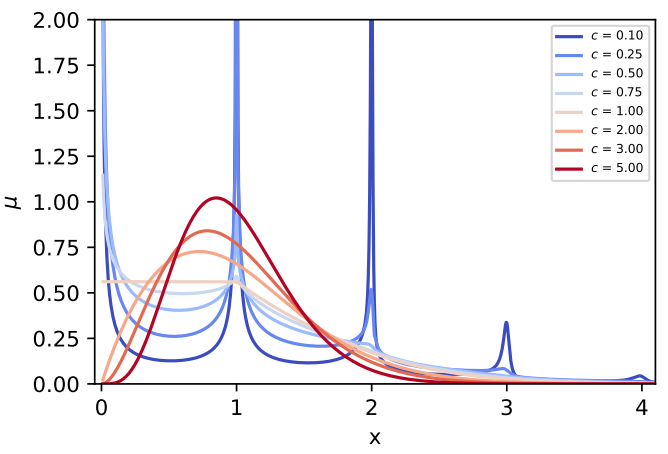

(a)

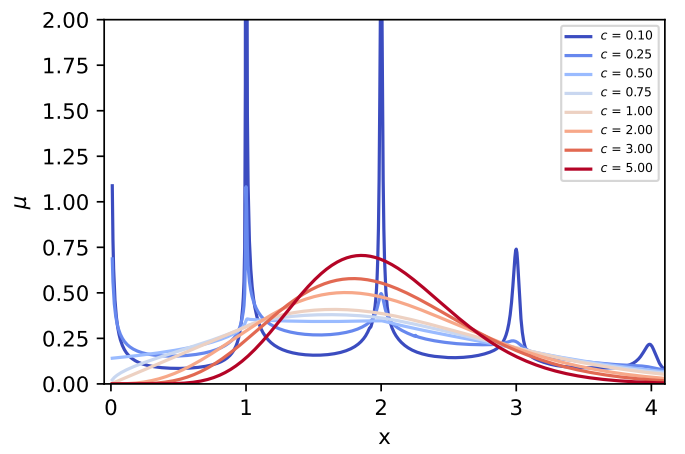

(b)

Figure 4: Plots of the Markov-Krein transforms of the limiting distributions of the Poisson limit theorem with parameters $a=1, \lambda=1$ in (a), $\lambda=2$ in (b), for different values of $c$.

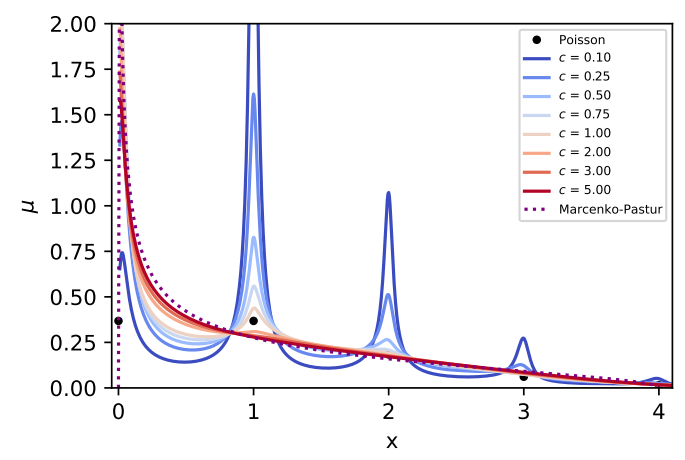

(a)

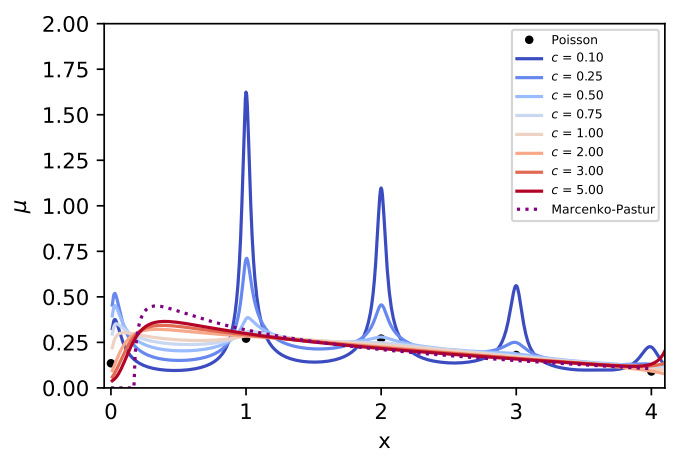

(b)

Figure 5: Plots of the numerical approximation of the limiting distribution of the Poisson limit theorem with parameters $a=1, \lambda=1$ in (a), $\lambda=2$ in (b), for different values of $c$. compared to the classical (Poisson) and free (Marčenko-Pastur) limiting distributions.

Since the distribution $v$ has support $\mathbb{R}_{+}$, we can take the inverse Laplace transform of the moment generating function evaluated at $-t$ :

$$
v(x)=\mathcal{L}_{t}^{-1}\left[\exp \left\{-a \lambda t_{2} F_{2}(\{1,1\},\{2, c+1\} ;-t)\right\}\right](x) .
$$

We can compute numerically $v$ using this last formula. We have plotted the distribution for different values in Fig 4.

The $c$-Poisson is then approximated numerically and we have plotted the different results in Fig 5.

c-cumulants: Using (175) and (156), we have that the $c$-cumulants of the $c$-Poisson are given by:

$$
\kappa_{k}^{(c)}=a^{k} \lambda,
$$

which is again expected for the limiting distribution of a Poisson limit theorem. 


\section{Conclusion}

In this note we have constructed the $c$-convolution, a one-parameter interpolation between the classical $(c=0)$ and the free $(c \rightarrow \infty)$ convolutions. Our main object of study is the HCIZ integral in the high temperature regime $\frac{N \beta}{2} \rightarrow c$, which is multiplicative for this convolution. It turns out that in this regime the HCIZ integral is the moment generating function of the so-called Markov-Krein transform of the distribution of interest so that the $c$-convolution of two distributions corresponds to a classical convolution of their Markov-Krein transforms. We finish this note with remarks and open questions that we believe are worth mentioning:

- We have not proved that the $c$-convolution preserves positivity and it is therefore possible that the $c$-convolution of two probability distributions is not a probability distribution. This is, however, unlikely since as pointed out in [51], if the positivity conjecture for Bessel functions is verified, then the c-convolution is well-defined. More generally, it will be interesting to know if one can (for a given $c$ or better independently of $c$ ) restrict the set of probability distributions so that the $c$-convolution is stable for this restricted set. In fact (133) at $c=1$ is satisfied for log-concave distributions and since this set is stable by classical convolution, we have that the set of continuous probability distributions whose Markov-Krein transforms are $\log$-concave is $c=1$-stable.

- If one can find such a restricted set it will be interesting to know if it is possible to construct a random object (such as an infinite random matrix) associated to a measure belonging to this restricted set, together with a certain notion of $c$-independence, such that the $c$-convolution of measures would correspond to a sum of those $c$-independent random objects.

- Another interesting and open direction of research is to know whether one can simplify the combinatorial formula of the moment-c-cumulant relations so that it can be expressed as a sum of $c$-weighted combinatorial objects, such as diagrams.

- In a previous note [52], we have introduced the multiplicative counterpart of the rank one HCIZ whose asymptotics is governed by the logarithm of the so-called $S$-transform of free probability (see also [41] for a similar rigorous derivation at $\beta=2$ ). The formula in [52] suggests that we can operate a similar construction yielding a multiplicative $c$ convolution that interpolates between the classical multiplicative convolution and the free multiplicative convolution. We leave this problem for future work.

\section{Acknowledgements}

The authors would like to thank Satya N. Majumdar and Jean-Philippe Bouchaud for many useful discussions and Florent Benaych-Georges for pointing out the relation between the positivity conjecture of Bessel functions and the $c$-convolution. P.M. would also like to thank D. Perales for indicating how to recover the combinatorial formula for the finite free cumulants from the combinatorics of the $c$-cumulants. The authors are grateful to the two anonymous referees for comments that have improve the overall clarity of the paper. 


\section{References}

[1] M. Potters and J.-P. Bouchaud, A first course in random matrix theory, Cambridge University Press, Cambridge, UK, ISBN 9781108768900 (2020), doi:10.1017/9781108768900.

[2] D. Voiculescu, Limit laws for random matrices and free products, Invent. Math. 104, 201 (1991), doi:10.1007/bf01245072.

[3] A. Nica and R. Speicher, Lectures on the combinatorics of free probability, Cambridge University Press, Cambridge, UK, ISBN 9780511735127 (2006), doi:10.1017/cbo9780511735127.

[4] F. Hiai and D. Petz, The semicircle law, free random variables and entropy (Mathematical Surveys \& Monographs), American Mathematical Society, USA, ISBN 0821841351 (2006).

[5] R. Speicher, On universal products, Fields Inst. Commun. 12, 257 (1997).

[6] R. Speicher, Boolean convolution, Free Probab. Theory (1997).

[7] N. Muraki, The five independences as natural products, Infin. Dimens. Anal. Quantum. Probab. Relat. Top. 06, 337 (2003), doi:10.1142/s0219025703001365.

[8] A. Nica, A one-parameter family of transforms, linearizing convolution laws for probability distributions, Commun. Math. Phys. 168, 187 (1995), doi:10.1007/BF02099588.

[9] M. Anshelevich, Partition-dependent stochastic measures and q-deformed cumulants, Doc. Math. 6, 343 (2001).

[10] F. Oravecz, Nica's q-convolution is not positivity preserving, Commun. Math. Phys. 258, 475 (2005), doi:10.1007/s00220-005-1307-8.

[11] F. Benaych-Georges and T. Lévy, A continuous semigroup of notions of independence between the classical and the free one, Ann. Probab. 39, 904 (2011), doi:10.1214/10-AOP573.

[12] R. Allez, J.-P. Bouchaud and A. Guionnet, Invariant beta ensembles and the Gauss-Wigner crossover, Phys. Rev. Lett. 109, 094102 (2012), doi:10.1103/PhysRevLett.109.094102.

[13] R. Allez, J.-P. Bouchaud, S. N. Majumdar and P. Vivo, Invariant $\beta$-Wishart ensembles, crossover densities and asymptotic corrections to the Marčenko-Pastur law, J. Phys. A: Math. Theor. 46, 015001 (2012), doi:10.1088/1751-8113/46/1/015001.

[14] K. D. Trinh and T. Shirai, The mean spectral measures of random Jacobi matrices related Gaussian beta ensembles, Electron. Commun. Probab. 20, 1 (2015), doi:10.1214/ECP.v20-4252.

[15] K. D. Trinh, Global spectrum fluctuations for Gaussian beta ensembles: A Martingale approach, J Theor Probab 32, 1420 (2017), doi:10.1007/s10959-017-0794-9.

[16] F. Nakano and K. D. Trinh, Gaussian beta ensembles at high temperature: Eigenvalue fluctuations and bulk statistics, J. Stat. Phys. 173, 295 (2018), doi:10.1007/s10955-018-21319.

[17] H. D. Trinh and K. D. Trinh, Beta Laguerre ensembles in global regime, Osaka J. Math. 58, 435, arXiv:1907.12267

[18] A. W. Marcus, Polynomial convolutions and (finite) free probability, arXiv:1504.00350. 
[19] A. W. Marcus, D. Spielman and N. Srivastava, Finite free convolutions of polynomials, arXiv:1504.00350.

[20] Harish-Chandra, Differential operators on a semisimple Lie algebra, Am. J. Math. 79, 87 (1957), doi:10.2307/2372387.

[21] C. Itzykson and J. -B. Zuber, The planar approximation. II, J. Math. Phys. 21, 411 (1980), doi:10.1063/1.524438.

[22] R. Coquereaux and J. B. Zuber, The Horn problem for real symmetric and quaternionic self-dual matrices, Sym. Integr. Geom. Meth. Appl. 15, 029 (2019), doi:10.3842/sigma.2019.029.

[23] J.-B. Zuber, Horn's problem and Harish-Chandra's integrals. Probability density functions, Ann. Inst. Henri Poincaré Comb. Phys. Interact. 5, 309 (2018), doi:10.4171/aihpd/56.

[24] R. Coquereaux, C. McSwiggen and J.-B. Zuber, Revisiting Horn's problem, J. Stat. Mech. 094018 (2019), doi:10.1088/1742-5468/ab3bc2.

[25] A. Guionnet and M. Maïda, Large deviations for the largest eigenvalue of the sum of two random matrices, Electron. J. Probab. 25, 1 (2020), doi:10.1214/19-EJP405.

[26] V. Gorin and A. W. Marcus, Crystallization of random matrix orbits, Int. Math. Res. Not. 883 (2018), doi:10.1093/imrn/rny052.

[27] I. P. Goulden, M. Guay-Paquet and J. Novak, Monotone Hurwitz numbers and the HCIZ integral, Ann. Math. Blaise Pascal 21, 71 (2014), doi:10.5802/ambp.336.

[28] J. Novak, On the complex asymptotics of the HCIZ and BGW integrals, arXiv:2006.04304.

[29] M. Bauer, D. Bernard and T. Jin, Universal fluctuations around typicality for quantum ergodic systems, Phys. Rev. E 101, 012115 (2020), doi:10.1103/PhysRevE.101.012115.

[30] B. Eynard, T. Kimura and S. Ribault, Random matrices, Saclay, Lecture Notes.

[31] I. G. Macdonald, Symmetric functions and Hall polynomials (Oxford classic texts in the physical sciences: Oxford mathematical mongraphs), Oxford University Press, Oxford, UK, ISBN 0198739125 (2015).

[32] R. P. Stanley, Some combinatorial properties of Jack symmetric functions, Adv. Math. 77, 76 (1989), doi:10.1016/0001-8708(89)90015-7.

[33] A. Matytsin, On the large-N limit of the Itzykson-Zuber integral, Nucl. Phys. B 411, 805 (1994), doi:10.1016/0550-3213(94)90471-5.

[34] A. Guionnet and O. Zeitouni, Large deviations asymptotics for spherical integrals, J. Funct. Anal. 188, 461 (2002), doi:10.1006/jfan.2001.3833.

[35] J. Bun, J. P. Bouchaud, S. N. Majumdar and M. Potters, Instanton approach to large N Harish-Chandra-Itzykson-Zuber integrals, Phys. Rev. Lett. 113, 070201 (2014), doi:10.1103/physrevlett.113.070201.

[36] E. Brézin and S. Hikami, Random matrix theory with an external source, Springer Singapore, ISBN 9789811033155 (2016), doi:10.1007/978-981-10-3316-2.

[37] P. J. Forrester, Probability densities and distributions for spiked and general variance Wishart $\beta$-ensembles, Random Matrices: Theory Appl. 2, 1350011 (2013), doi:10.1142/S2010326313500111. 
[38] A. W. Marcus and W. Yomjinda, Analysis of rank 1 perturbations in general $\beta$ ensembles (2016), https://web.math.princeton.edu/ amarcus/papers/beta.pdf.

[39] E. Marinari, G. Parisi and F. Ritort, Replica field theory for deterministic models. II. A non-random spin glass with glassy behaviour, J. Phys. A: Math. Gen. 27, 7647 (1994), doi:10.1088/0305-4470/27/23/011.

[40] A. Guionnet, A Fourier view on the R-transform and related asymptotics of spherical integrals, J. Funct. Anal. 27, 7647 (1994), doi:10.1088/0305-4470/27/23/011.

[41] V. Gorin and Y. Sun, Gaussian fluctuations for products of random matrices, arXiv:1812.06532.

[42] O. Arizmendi and D. Perales, Cumulants for finite free convolution, J. Comb. Theory, Series A 155, 244 (2018), doi:10.1016/j.jcta.2017.11.012.

[43] J. Baik, E. Collins-Woodfin, P. Le Doussal and H. Wu, Spherical spin glass model with external field, J. Stat. Phys. 183, 31 (2021), doi:10.1007/s10955-021-02757-7.

[44] S. Kerov, Interlacing measures, Amer. Math. Soc. Transl. 181, 35 (1998).

[45] J. Faraut and F. Fourati, Markov-Krein transform, Coll. Math. 144, 137 (2016).

[46] N. Lid Hjort and A. Ongaro, Exact inference for random Dirichlet means, Stat. Infer. Stoch. Proc. 8, 227 (2005), doi:10.1007/s11203-005-6068-7.

[47] D. Michele Cifarelli and E. Regazzini, Distribution functions of means of a Dirichlet process, Ann. Statist. 18, 429 (1990), doi:10.1214/aos/1176347509.

[48] G. Letac and M. Piccioni, Dirichlet curves, convex order and Cauchy distribution, Bernoulli 24, 1 (2018), doi:10.3150/15-BEJ765.

[49] R. Askey and J. Wimp, Associated Laguerre and Hermite polynomials, Proc. R. Soc. Edinb.: Math. 96, 15 (1984), doi:10.1017/S0308210500020412.

[50] F. Lehner, Cumulants in noncommutative probability theory I. Noncommutative exchangeability systems, Math. Z. 248, 67 (2004), doi:10.1007/s00209-004-0653-0.

[51] F. Benaych-Georges, C. Cuenca and V. Gorin, Matrix addition and the Dunkl transform at high temperature, arXiv:2105.03795.

[52] P. Mergny and M. Potters, Asymptotic behavior of the multiplicative counterpart of the Harish-Chandra integral and the S-transform, arXiv:2007.09421. 